\title{
Latent hardening size effect in small-scale plasticity
}

\author{
Lorenzo Bardella ${ }^{1}$, Javier Segurado ${ }^{2,3}$, Andrea Panteghini ${ }^{1}$ and \\ Javier Llorca ${ }^{2,3}$
}

\begin{abstract}
We aim at understanding the multislip behaviour of metals subject to irreversible deformations at small-scales. By focusing on the simple shear of a constrained single-crystal strip, we show that discrete Dislocation Dynamics (DD) simulations predict a strong latent hardening size effect, with smaller being stronger in the range $[1.5 \mu \mathrm{m}, 6 \mu \mathrm{m}]$ for the strip height. We attempt to represent the DD pseudo-experimental results by developing a flow theory of Strain Gradient Crystal Plasticity (SGCP), involving both energetic and dissipative higher-order terms and, as a main novelty, a strain gradient extension of the conventional latent hardening. In order to discuss the capability of the SGCP theory proposed, we implement it into a Finite Element (FE) code and set its material parameters on the basis of the DD results. The SGCP FE code is specifically developed for the boundary value problem under study so that we can implement a fully implicit (Backward Euler) consistent algorithm. Special emphasis is placed on the discussion of the role of the material length scales involved in the SGCP model, from both the mechanical and numerical points of view.
\end{abstract}

(Some figures may appear in colour only in the online journal)

\section{Introduction}

This work focuses on the multislip behaviour of a single-crystal metal strip sheared between two bodies impenetrable to dislocations. On the basis of this benchmark, we analyze the capability of a Strain Gradient Crystal Plasticity (SGCP) model to reproduce the fundamental features of the strip behaviour by comparison with the pseudo-experimental results obtained from two-dimensional discrete Dislocation Dynamics (DD) simulations. We are particularly interested in analyzing the effect of the strip size on its mechanical response. 


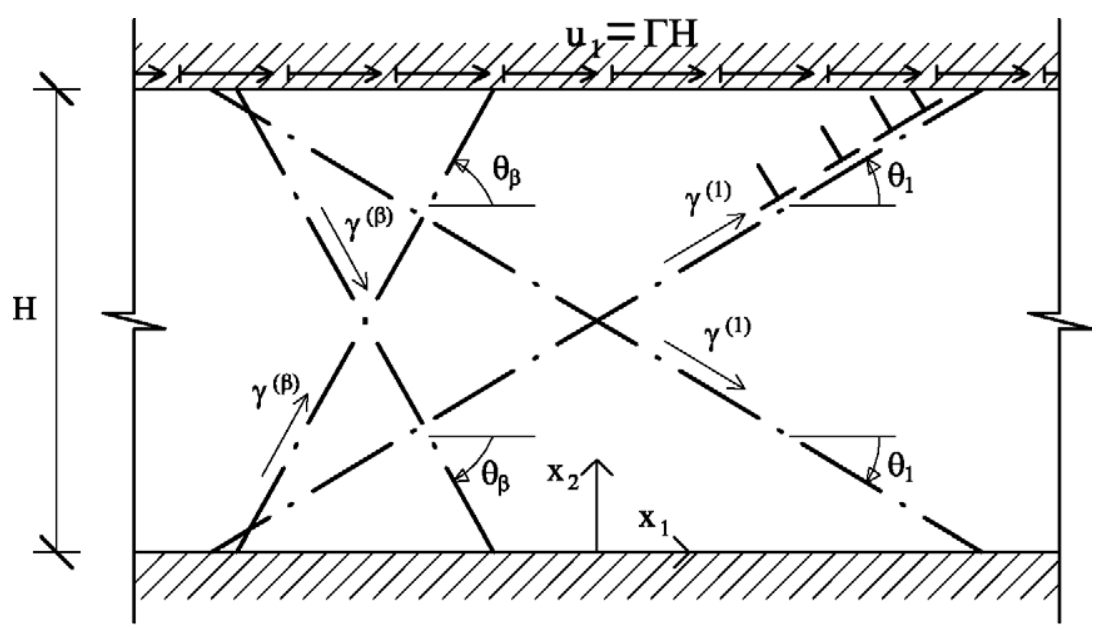

Figure 1. Simple shear of a constrained crystalline strip with multiple symmetric couples of slip systems.

The details of the DD code here employed are reported in $[1,2]^{4}$. The SGCP model consists of an extension of the model developed in [5-7] (see also Gurtin et al [8,9]): it is of the higherorder and work-conjugate type (see, e.g., [10] and references therein) and it involves both energetic and dissipative higher-order terms; in the limit of vanishing material length scales, the SGCP model particularizes to the crystal viscoplasticity model of Peirce $e t$ al [11], within the small strain range 5 . Within the strain gradient plasticity framework, rate-dependence provides two benefits: (i) it is not necessary to impose higher-order boundary conditions on the internal boundaries between elastic and plastic regions, and (ii) more efficient algorithms can be developed to obtain numerical solutions. Moreover, viscoplasticity particularizes to the rate-independent case for an appropriate limit choice of a rate sensitivity material parameter.

In this work, the results obtained from DD and SGCP are analyzed and discussed with particular emphasis on the role of the material length scales involved in the latter.

First, the effect of the strip height has been analyzed by means of DD simulations for a crystal endowed, in each material point, with a single couple of active slip systems symmetrically inclined with respect to the applied shear direction (see figure 1). We observe a size effect in the mechanical behaviour for strip heights in the range $1.5-6 \mu \mathrm{m}$. The size effect consists of an increase in strain hardening with diminishing size, without strengthening (i.e. an increase in what is recognized as the initial yield stress). This effect can be reproduced both qualitatively and quantitatively by using the SGCP model with the energetic material length scale set to $\ell=0.6 \mu \mathrm{m}$.

Second, the latent hardening has been studied by introducing a further couple of active slip systems symmetrically inclined, with respect to the applied shear direction, by a larger angle than that characterizing the first couple. The DD results show a much stiffer response in the crystals endowed with two pairs of systems at a strain larger than about twice the strain corresponding to the initial yield stress, the latter still being independent of the size. This indicates a large latent hardening with related the size of effect 'smaller being stronger'. In

\footnotetext{
4 Among the seminal works on DD let us mention those of Kubin and co-workers (see, e.g., [3]) and van der Giessen and Needleman [4].

5 Borg [12] also extended the conventional crystal plasticity of Peirce et al [11] to the strain gradient case, but, contrary to the modelling proposed here, without including the energetic higher-order terms and by maintaining the conventional equations for the latent hardening.
} 
order to describe this effect by means of SGCP, we propose a strain gradient extension of the conventional latent hardening of Peirce $\mathrm{et} \mathrm{al}[11]^{6}$. This constitutes a further source of coupling between the extended Schmid equations governing the flow on the slip systems, with respect to the coupling provided in our SGCP by the definition of a defect energy as a function of Nye's dislocation density tensor (Nye [15], Fleck and Hutchinson [16], Gurtin [17], Bardella and Giacomini [7]).

Currently, the prediction of the strain hardening on the basis of the interaction among slip systems is one of the most relevant problems in small-scale plasticity (see, for instance, the DD analyses of Devincre $e t$ al [18]).

In order to compare discrete DD and SGCP, we have implemented the SGCP theory proposed here. In particular, we have developed a specific Finite Element (FE) algorithm for the simple shear benchmark of interest, for which a unidimensional finite element is adequate because of the chosen symmetry in the slip systems configuration. The FE degrees of freedom are the displacement and the plastic slips and the time integration consists of a fully implicit Backward Euler scheme. The SGCP model is implemented into a user element subroutine (uel) for the commercial FE code ABAQUS [19].

Brief literature survey of recent papers on $S G C P$, its comparison with $D D$, and its implementation. Among other SGCP theories, we find it worth mentioning the backstressbased SGCP theories developed by Yefimov et al [20] and Geers, Brekelmans, and co-workers [21-24]. These theories are characterized by the introduction into the Schmid law of a backstress contribution dependent on evolution equations for the dislocation densities, based on dislocation mechanics. Hence, these theories are closer to DD than the work-conjugate SGCP theory developed here; however, typically, the backstress-based SGCP theories only consider energetic strain gradient terms and when the interaction among slip systems is accounted for (see, e.g., [22]), which is central in this paper, it is uncertain whether or not such theories are compatible with thermodynamics (see $[10,25,26]$ ).

Yefimov et al $[20,27]$ verified that their backstress-based SGCP theory can represent the results obtained from DD simulations for a few meaningful boundary value problems in which the crystal is assumed to deform in single slip.

Among earlier comparisons between DD and SGCP we mention the works of Bittencourt et al [28] and Nicola et al [29], both concerned with testing Gurtin's 2002 SGCP theory [17]; such a theory involves only energetic higher-order terms and neglects the latent hardening. Nicola et al [29] exploited the problem of a thin strip on a semi-infinite substrate subject to thermal loading to identify the most appropriate form of the defect energy in Gurtin's 2002 model [17]. They considered a single couple of symmetric slip systems and concluded that the defect energy should be proportional, through appropriate material parameters, to the sum of the squared gradients of the plastic slips. Here, we choose to define the defect energy as a function of Nye's tensor (as originally proposed by Gurtin [17]), as this provides a natural source of interaction among slip systems, as pointed out in [7]. More recently, this higherorder coupling has been investigated also by Bargmann et al [30] within the context of a finite deformation SGCP theory. Regarding the numerical solution of the related boundary value problems, Bargmann et al [30] propose a dual-mixed FE implementation characterized by a mixed implicit-explicit time integration.

Reddy et al [31] propose an FE implementation of Gurtin's 2002 SGCP theory [17], assuming the defect energy proposed by Nicola et al [29], thus without interactions among

6 Among the fundamental studies on the conventional latent hardening we mention those of Franciosi et al [13] and Havner [14], and references therein. 
slip systems. Other features of this SGCP model are its rate independence with standard linear, isotropic hardening. Reddy et al [31] develop a weak formulation in which the primal unknowns are the displacements, the plastic slips, and the backstress related to the microstress (that is, the vectorial higher-order stress [32]). The algorithm associated with the weak formulation has the objective of designing efficient general purpose finite elements.

Wulfinghoff and Böhlke [33] propose a higher-order, work-conjugate SGCP theory involving, as an exclusive unconventional primal (kinematic) variable, the gradient of the sum of the plastic slip rates taken in modulus. They also consider a standard latent hardening into a Perzyna-like viscoplasticity framework. About the FE implementation, Wulfinghoff and Böhlke [33] write the variational statement in such a way as to impose in a weak form, through the use of a scalar micromorphic-like variable, the equality between a scalar Lagrange parameter and the microstress divergence ${ }^{7}$. This allows the addition of one sole extra degree of freedom, with respect to those involved in standard crystal viscoplasticity.

Other investigators (see, e.g., [10, 20, 35] and references therein) have developed staggered solution schemes with explicit time integration for general purpose finite elements having as nodal degrees of freedom the displacement plus some plasticity measure appropriate to the SGCP of concern.

Another strategy to obtain a robust general purpose $\mathrm{FE}$ algorithm for higher-order gradient plasticity consists of resorting to a discontinuous Galerkin scheme, as proposed by Ostien and Garikipati [36] in order to implement the phenomenological (i.e. neglecting the crystal lattice) gradient plasticity involving the plastic spin due to Gurtin [37]. The nonlinear problem is relaxed by defining some primal plasticity unknowns in average on the finite element, instead of defining them as nodal degrees of freedom. It is here of some relevance to note that Gurtin's 2004 gradient plasticity [37] has been shown to be the phenomenological theory closest to crystal plasticity when multislip occurs $[38,39]$.

Notation. We use lightface letters for scalars. For first-, second-, and third-order tensors we use boldface letters, with some exceptions where the use of indices makes the equations clearer. ' ' represents the scalar product (for instance, for vectors: $s=\boldsymbol{m} \cdot \boldsymbol{n} \equiv m_{i} n_{i}$, while, for second-order tensors: $\tau=\sigma \cdot \boldsymbol{\mu} \equiv \sigma_{i j} \mu_{i j}$ ); ' $\times$ ' is adopted for the vector product ( $\boldsymbol{t}=\boldsymbol{m} \times \boldsymbol{n} \equiv e_{i j k} m_{j} n_{k}=t_{i}$, with $e_{i j k}$ the alternating symbol); ' $\otimes$ ' is employed for the tensor product of vectors $\left(\boldsymbol{S}=\boldsymbol{m} \otimes \boldsymbol{n} \equiv m_{i} n_{j}=S_{i j}\right)$. For $\boldsymbol{\sigma}$ a second-order tensor and $\boldsymbol{n}$ a vector: $\boldsymbol{t}^{0}=\boldsymbol{\sigma n} \equiv \sigma_{i j} n_{j}=t_{i}^{0}$; for $\mathbb{L}$ a fourth-order tensor and $\varepsilon$ a second-order tensor: $\boldsymbol{\sigma}=\mathbb{L} \varepsilon \equiv L_{i j k l} \varepsilon_{k l}=\sigma_{i j}$. $\nabla \boldsymbol{u} \equiv \partial u_{i} / \partial x_{j} \equiv u_{i, j}$, div $\boldsymbol{\sigma} \equiv \sigma_{i j, j}, \operatorname{curl} \gamma \equiv e_{j k l} \gamma_{i l, k}$, and $\operatorname{sym} \boldsymbol{S} \equiv\left(S_{i j}+S_{j i}\right) / 2$ designate, respectively, the gradient of the vector field $\boldsymbol{u}$, the divergence of the second-order tensor $\sigma$, the curl of the second-order tensor $\gamma$, and the symmetric part of the second-order tensor $\boldsymbol{S}$. The number of slip systems available in the crystal is $A$ and they are labeled with lowercase Greek indices. The underline is used to indicate a vector of dimension $A$ (e.g. $\underline{\gamma}$ collects all the plastic slips).

The benchmark. We focus on the boundary value problem sketched in figure 1, consisting of the simple shear of a crystalline strip constrained between two bodies in which dislocations cannot penetrate, unbounded along $x_{1}$ and $x_{3}$, of height $H$ along the $x_{2}$-direction. This benchmark has been one of the most exploited in the literature to evaluate the capability of various models to describe the behaviour of miniaturized metals. In fact, it allows the

7 Earlier, a similar technique was proposed by Shu et al [34] within the context of the Toupin-Mindlin strain gradient theory. The relaxed version of the Principle of Virtual Work obtained by Shu et al [34] allowed them to use $C^{0}$-continuous finite elements. 
reproduction of one of the most fundamental deformation modes in small-scale plasticity in a very simple way. The crystal is characterized by (i) isotropic linear elasticity, with Young's modulus equal to $70 \mathrm{MPa}$ and Poisson's ratio equal to 0.33 , so that the shear modulus $\mu=26315 \mathrm{MPa}$, and (ii) $B$ couples of slip systems (the total number of systems is then $A=2 B$ ), each couple $\beta$ consisting of two possible glides symmetrically oriented with respect to any plane of constant $x_{2}$ by an angle $\theta_{\beta}$. The strip is sheared by applying to the plane $x_{2}=H$ a uniform displacement equal to $\Gamma H$ along the $x_{1}$-direction (so that $\Gamma$ is called the applied shear). The conventional boundary conditions are given in terms of displacement $\boldsymbol{u}$ and read

$$
\begin{array}{ll}
u_{1}\left(x_{2}=0\right)=0 ; & u_{1}\left(x_{2}=H\right)=\Gamma H, \\
u_{2}\left(x_{2}=0\right)=0 ; & u_{2}\left(x_{2}=H\right)=0 .
\end{array}
$$

Outline of the paper. In section 2 we describe the DD simulations and discuss their results. Section 3 is devoted to presenting the equations governing the SGCP model in the general three-dimensional case, and to its particularization to the benchmark of interest here. The consistent and implicit FE algorithm of this benchmark is described in detail in section 4. The results obtained with DD and SGCP are compared and discussed in section 5. Some concluding remarks and open issues are given in section 6 .

\section{Discrete Dislocation Dynamics simulations of the constrained simple shear}

The DD results have been obtained by means of the code developed as described in Segurado et al [1] (see also [2]). The method has been formerly established by Kubin and co-workers (see, e.g., [3]) and van der Giessen and Needleman [4]. The model basically describes plasticity as the irreversible motion of dislocations into a linear elastic crystal. Dislocation glide, in our plane strain benchmark, is governed by

$v_{\beta}^{i}=\frac{b}{B_{\text {drag }}}\left[\left(\frac{\hat{\sigma}_{22}-\hat{\sigma}_{11}}{2}+\sum_{j \neq i} \frac{\sigma_{22}^{j}-\sigma_{11}^{j}}{2}\right) \sin \left(2 \theta_{\beta}\right)+\left(\hat{\sigma}_{12}+\sum_{j \neq i} \sigma_{12}^{j}\right) \cos \left(2 \theta_{\beta}\right)\right]$

in which $v_{\beta}^{i}$ is the velocity of the $i$ dislocation on the $\beta$ slip system, $b$ is the Burgers vector magnitude, $B_{\text {drag }}$ is the drag coefficient, $\sigma^{i}$ is the stress field exerted by the $i$ dislocation in an unbounded medium, and $\hat{\sigma}$ is the stress due to both the applied load and the image tractions applied at the boundary to make up for the use of the dislocation stress fields of the unbounded medium. At each time step along the loading history, $\hat{\sigma}$ is obtained by means of a linear elastic $\mathrm{FE}$ analysis.

A random distribution of sources is set in the strip such that in any source a dislocation dipole nucleates when the resolved shear stress reaches the nucleation stress for a certain minimum lapse of time, called the nucleation time. Dislocations of opposite sign gliding on the same slip system are annihilated when they are closer than the so-called annihilation length. Dislocation pinning by obstacles is accounted for by a random distribution of obstacles where dislocations get blocked if their driving shear stress is lower than the obstacle strength.

The parameters involved in the simulation are set as follows: $b=0.25 \mathrm{~nm}, B_{\text {drag }}=$ 1.E $-4 \mathrm{Ns} \mathrm{m}^{-2}$, source density $=42 \mu \mathrm{m}^{-2}$, obstacle density $=126 \mu \mathrm{m}^{-2}$, nucleation stress $=25 \mathrm{MPa}$ (with a standard deviation of $5 \mathrm{MPa}$ ), nucleation time $=0.01 \mu \mathrm{s}$, mean nucleation distance $=0.0625 \mu \mathrm{m}$, obstacle strength $=300 \mathrm{MPa}$, annihilation length $=6 b$ and slip system spacing $=100 \mathrm{~b}$. Such values agree with those used in previous DD simulations $[40,41]$. 


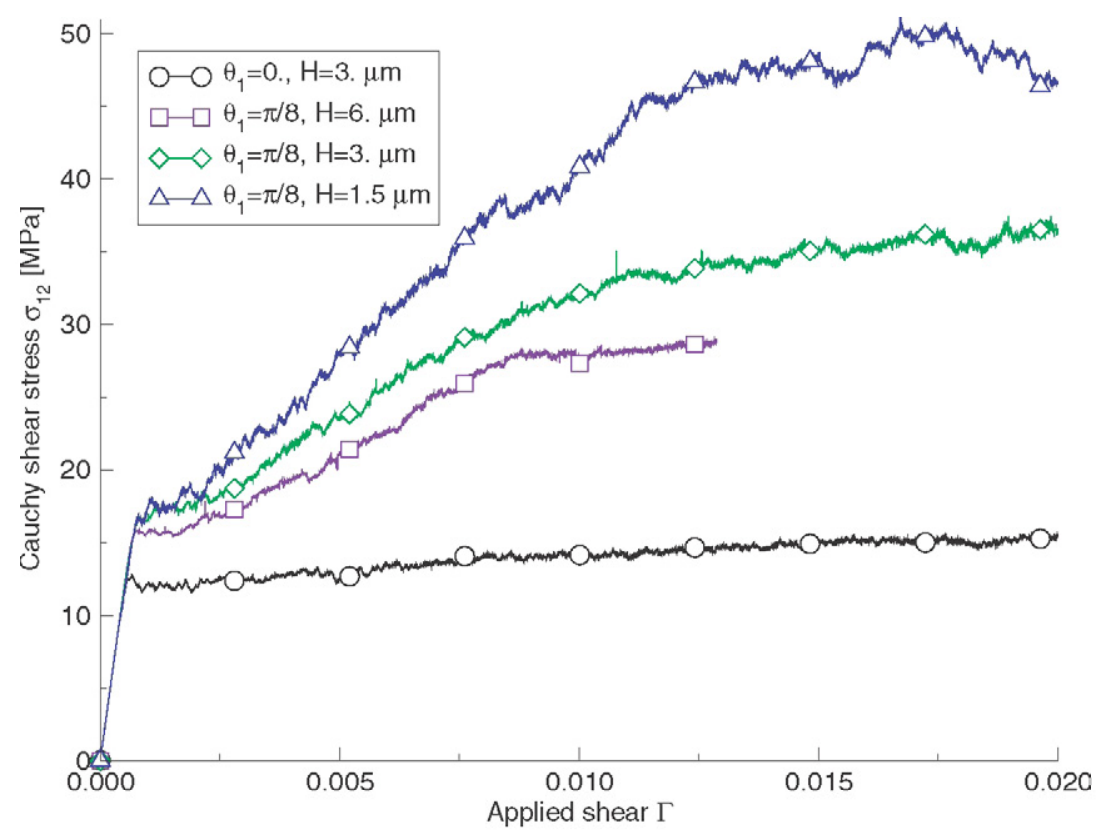

Figure 2. Results of DD simulations on a constrained crystalline strip with a single couple of symmetric slip systems.

The inclination angles of the two couples of slip systems considered, with respect to the applied shear direction, are $\pm \pi / 8$ and $\pm \pi / 5$.

The total time of the simulation is $1 . \mathrm{E}-5 \mathrm{~s}$, while the time integration step is $5 . \mathrm{E}-11 \mathrm{~s}$, so that each analysis involves $2 . \mathrm{E}+5$ integration steps.

The length of the strip is $10 \mathrm{H} / 3$, in such a way that the results be marginally affected by the behaviour at the boundaries orthogonal to the shear direction, where dislocations are free to exit the strip. Longer strips would require too large of a computational effort.

\subsection{The results of $D D$ simulations}

The results of the DD analyses are reported in figures 2-4. Each plot is obtained by the average of three analyses run with the same initial data, but different realization, e.g., in terms of position of sources and obstacles (the results may also slightly differ because of the chaotic behaviour inherent to discrete DD).

Varying the total time of the analysis (the explored range is [5.E $-6 \mathrm{~s}, 2 . \mathrm{E}-5 \mathrm{~s}]$ ) has given a negligible effect on the stress averages we are interested in, so that we conclude that DD simulations are quite rate insensitive.

In the case in which one sole couple of slip systems is considered the results of figure 2 show that, within the range of about $\Gamma \in[0,0.01]$, decreasing the height $H$ leads to an increase in strain hardening, while no strengthening is observed. All the analyses are run until the applied strain is $\Gamma=2 \%$, with the exception of the analysis for the largest strip ( $H=6 \mu \mathrm{m})$, ended at a strain of about $1.26 \%$ because the DD simulation is too much expensive for such a large sample. The applied shear amplitude considered is well representative of the relevant results obtainable by means of the DD here employed; in fact, soon after the maximum applied shear considered, the DD response reaches an almost steady-state behaviour, corresponding to the maximum (plateau) value of dislocations present in the strip. 


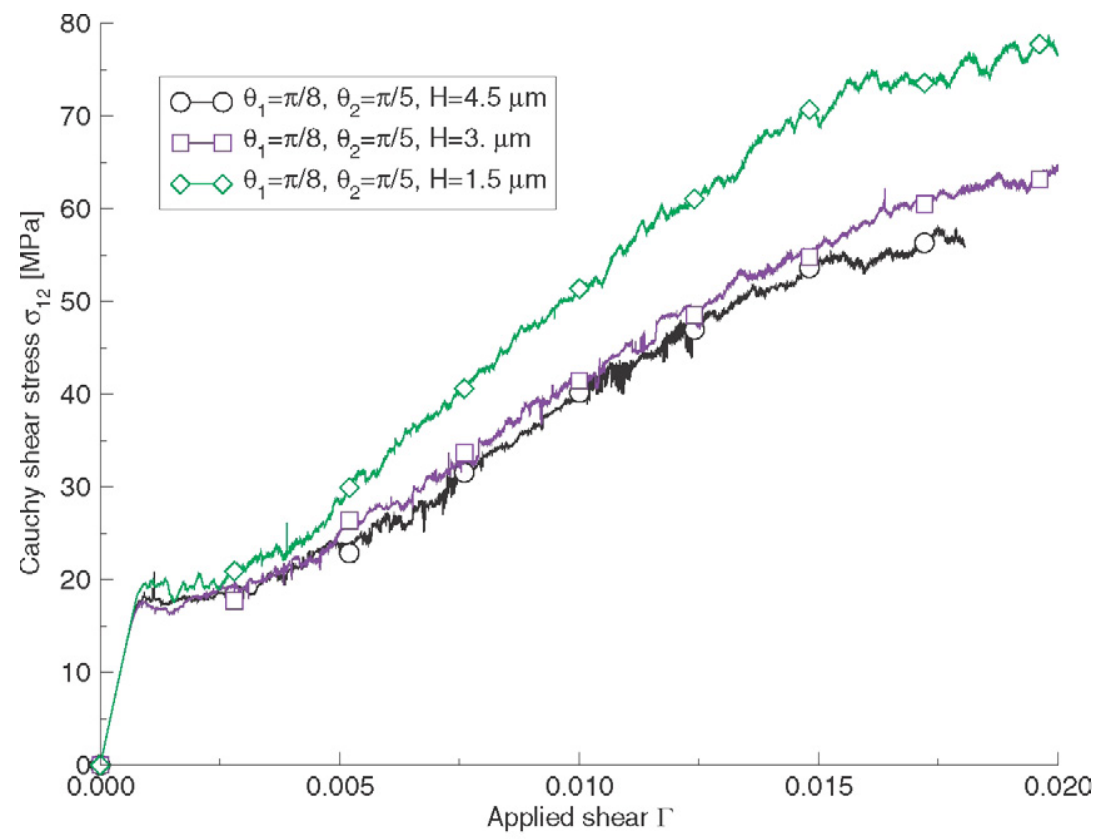

Figure 3. Results of DD simulations on a constrained crystalline strip with two couples of symmetric slip systems.

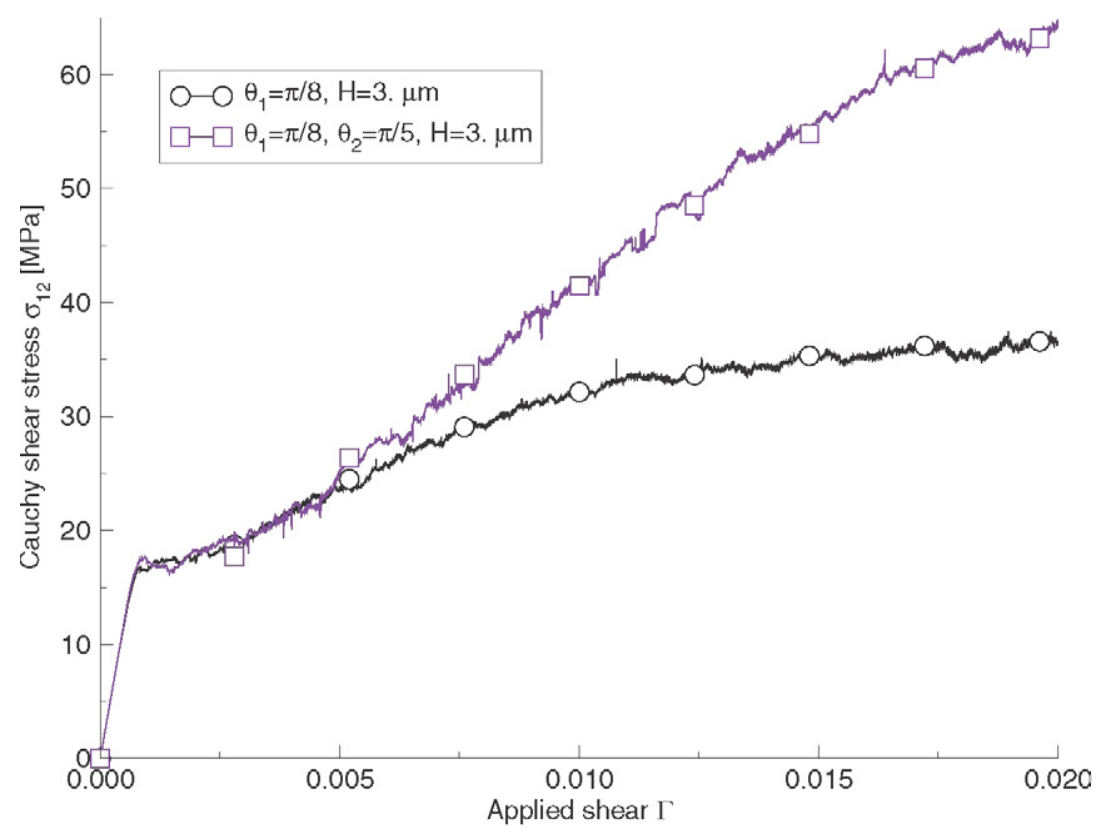

Figure 4. Results of DD simulations on a constrained crystalline strip of size $H=3 \mu \mathrm{m}$ endowed with either one couple or two couples of symmetric slip systems. 
The minimum strip height considered here is $H=1.5 \mu \mathrm{m}$. As pointed out by Nicola et al [29] and Segurado and Llorca [41], the origin of the hardening predicted by DD in smaller specimens may be nucleation controlled to a large extent. Such a phenomenon would be out of the description capability of the SGCP model considered in this investigation.

The addition of a second couple of systems, see figure 3 , provides an extremely high latent hardening, as, for each size $H$, the stress-strain curve is higher than that with one sole couple for about the whole range of applied shear $\Gamma$, as shown in figure 4 for the case $H=3 \mu \mathrm{m}$. This means that the compliance introduced by the possibility of further slip given by the second couple of systems is overcome by the obstruction to glide provided on the first (less inclined) couple of systems by dislocations nucleating on the second couple.

Since the analyses with two couples of slip systems are numerically more expensive than those in which the crystallography consists of a single couple of systems, the biggest strip in the case of two couples has $H=4.5 \mu \mathrm{m}$, smaller that the biggest strip with one sole couple. Also the analysis for the biggest strip endowed with two couples ended before $\Gamma$ reached the value of $2 \%$.

The slight softening behaviour observable within a small strain range after the yield stress is reached may be ascribed to the sudden and simultaneous activation of several sources, to which a conspicuous plastic strain and a stress decay correspond. Then, dislocations start to pile up at the impenetrable boundaries and only a few sources remain (or become) active, leading to what is macroscopically recognized as hardening, until a maximum plateau stress is reached, corresponding to a steady-state dislocation density.

\section{The Strain Gradient Crystal Plasticity (SGCP) model}

The SGCP model here presented, aiming at describing the behaviour observed in the DD simulations, is an extension of the model developed in Bardella [5,6], Gurtin et al [8,9], and Bardella and Giacomini [7]. The resulting SGCP model can also be seen as a strain gradient extension of the classical crystal viscoplasticity of Peirce et al [11] particularized to the small strain regime.

\subsection{Governing equations for general three-dimensional boundary value problems}

In the general case, we are concerned with the mechanical response of a single crystal occupying a space region $\Omega$, whose external surface $S$, of outward normal $\boldsymbol{n}$, consists of two couples of complementary parts: the first couple consists of $S_{t}$, where the conventional tractions $t^{0}$ are known, and $S_{u}$, where the displacement $\boldsymbol{u}^{0}$ is known, whereas the second couple consists of $S_{t}^{\text {dis }}$, where dislocations are free to exit the body, and $S_{u}^{\mathrm{dis}}$ where dislocations are blocked. $S=S_{t} \cup S_{u}=S_{t}^{\text {dis }} \cup S_{u}^{\text {dis }}$. The crystal is free from body forces.

We limit the theoretical part to the presentation of the compatibility, balance, and constitutive equations. The constitutive equations (given in section 3.1.3) include the novel latent hardening strain gradient extension. For the derivation of and more insight on the other governing equations presented next, the reader is referred to [5-7, 9, 39].

\subsubsection{Compatibility equations.}

Field equations. $\quad \varepsilon$ is the total strain, defined in terms of the displacement $\boldsymbol{u}$ as

$$
\varepsilon=\operatorname{sym} \nabla \boldsymbol{u} .
$$


The plastic part of the displacement gradient (i.e. the plastic distortion) reads

$$
\gamma=\sum_{\beta=1}^{A} \gamma^{(\beta)} \boldsymbol{m}^{(\beta)} \otimes \boldsymbol{n}^{(\beta)}=\sum_{\beta=1}^{A} \gamma^{(\beta)} \boldsymbol{S}^{(\beta)},
$$

in which, for its system $\beta, \gamma^{(\beta)}$ is the plastic slip, $\boldsymbol{m}^{(\beta)}$ and $\boldsymbol{n}^{(\beta)}$ are unit vectors defining the slip direction and the slip plane normal, respectively, and $S^{(\beta)}=\boldsymbol{m}^{(\beta)} \otimes n^{(\beta)}$ is the Schmid tensor.

$$
\boldsymbol{\varepsilon}^{p}=\operatorname{sym} \gamma=\sum_{\beta=1}^{A} \gamma^{(\beta)} \boldsymbol{\mu}^{(\beta)}
$$

is the plastic strain, where $\boldsymbol{\mu}^{(\beta)}=\operatorname{sym} \boldsymbol{S}^{(\beta)}$. Nye's dislocation density tensor $\boldsymbol{\alpha}$ (Nye [15]) is a representation of Geometrically Necessary Dislocations (GNDs) $)^{8}$ such that $\alpha_{i j}$ is the $i$-component of the resultant Burgers vector related to GNDs of line vector $j$. Nye's tensor represents the incompatibility of the plastic deformation field, dependent on densities of excess dislocations, i.e. GNDs. A definition suitable for developing a mechanical theory within a continuum mechanics framework (Fleck and Hutchinson [16]) reads:

$$
\alpha=\operatorname{curl} \gamma
$$

After inserting definition (3.1) into (3.2), one obtains Nye's dislocation density tensor in terms of the slip gradients in the slip direction (interpretable as a density of pure edge dislocations $\rho_{\perp}^{(\beta)}=\nabla \gamma^{(\beta)} \cdot \boldsymbol{m}^{(\beta)}$ lying along the transverse direction $\boldsymbol{t}^{(\beta)}=\boldsymbol{m}^{(\beta)} \times \boldsymbol{n}^{(\beta)}$ ) and in the transverse direction (corresponding to a density of pure screw dislocations $\rho_{\odot}^{(\beta)}=-\nabla \gamma^{(\beta)} \cdot \boldsymbol{t}^{(\beta)}$ lying along the slip direction):

$$
\alpha=\sum_{\beta=1}^{A} \boldsymbol{m}^{(\beta)} \otimes\left(\rho_{\perp}^{(\beta)} \boldsymbol{t}^{(\beta)}+\rho_{\odot}^{(\beta)} \boldsymbol{m}^{(\beta)}\right) .
$$

By defining the third-order tensor

$$
\boldsymbol{N}^{(\beta)}=\boldsymbol{m}^{(\beta)} \otimes\left(\boldsymbol{m}^{(\beta)} \otimes \boldsymbol{t}^{(\beta)}-\boldsymbol{t}^{(\beta)} \otimes \boldsymbol{m}^{(\beta)}\right)
$$

Nye's tensor can also be written by singling out its dependence on the plastic slip gradient [5]:

$$
\alpha_{i j}=\sum_{\beta=1}^{A} \gamma_{, k}^{(\beta)} N_{i k j}^{(\beta)} .
$$

We also define the projection of the plastic slip gradient onto the normal to the slip plane as

$$
\rho_{N}^{(\beta)}=\nabla \gamma^{(\beta)} \cdot \boldsymbol{n}^{(\beta)} .
$$

Kinematic boundary conditions.

$$
u=u^{0} \quad \text { on } S_{u} \quad \text { and } \quad \gamma^{(\beta)}=0 \quad \forall \beta \quad \text { on } S_{u}^{\text {dis }} .
$$

8 About the role of GNDs in small-scale plasticity, see, e.g., Ashby [42], Fleck and Hutchinson [16], Arsenlis and Parks [43], Needleman and Sevillano [44], and Kysar et al [45]. 


\subsubsection{Balance equations.}

Field equations. The absence of body forces allows the standard balance equation to be written as

$$
\operatorname{div} \sigma=\mathbf{0} \quad \text { in } \Omega
$$

with $\sigma$ the Cauchy stress. The unconventional (higher-order) balance equations read:

$$
s^{(\beta)}-\tau^{(\beta)}-\operatorname{div} \boldsymbol{\xi}^{(\beta)}=0 \quad \forall \beta \text { in } \Omega,
$$

where

$$
\tau^{(\beta)}=\sigma \cdot \mu^{(\beta)}
$$

is the resolved (Schmid) stress,

$$
\xi_{i}^{(\beta)}=d_{k l} N_{k i l}^{(\beta)}+\omega_{\perp}^{(\beta)} m_{i}^{(\beta)}-\omega_{\odot}^{(\beta)} t_{i}^{(\beta)}+\omega_{N}^{(\beta)} n_{i}^{(\beta)} \quad \forall \beta
$$

is the so-called microstress [7,17], in which $d$ is the defect stress, conjugate to Nye's tensor in the internal work, $s^{(\beta)}$ is the slip resistance, conjugate to the plastic slip rate $\dot{\gamma}^{(\beta)}$ in the internal work, and $\omega_{\perp}^{(\beta)}, \omega_{\odot}^{(\beta)}, \omega_{N}^{(\beta)}$ are higher-order dissipative stresses, conjugate to the rates $\dot{\rho}_{\perp}^{(\beta)}, \dot{\rho}_{\odot}^{(\beta)}, \dot{\rho}_{N}^{(\beta)}$, respectively, in the internal work.

It is important to notice that the definition of dissipative higher-order stresses $\omega_{I}^{(\beta)}$ work-conjugate to $\dot{\rho}_{I}^{(\beta)}(I=\perp, \odot, N)$ is known to deliver the capability to describe some strengthening accompanied with diminishing size [5]. Here, even though the DD results presented in section 2 show a negligible strengthening, we still consider a SGCP theory involving the dissipative higher-order stresses $\omega_{I}^{(\beta)}$. In fact, this provides a few benefits to the modelling. First, it allows the use of each plastic slip component as a primal (kinematic) variable, with corresponding nodal degrees of freedom in the FE formulation. Otherwise, the exclusive inclusion of Nye's tensor as higher-order primal variable would make the definition of the higher-order boundary conditions more convoluted (see $[5,46]$ ) and, consequently, the FE formulation also. Second, the presence of dissipative higher-order stresses mitigates the problems of convergence of the FE implicit algorithm; of course, in order to represent the DD results, we will appropriately choose the relevant material parameters: this will turn out in 'dissipative length scales' significantly smaller than the 'energetic length scale', the latter governing the influence of the defect stress.

Static boundary conditions.

$$
\boldsymbol{\sigma} \boldsymbol{n}=\boldsymbol{t}^{0} \quad \text { on } S_{t} \quad \text { and } \quad \boldsymbol{\xi}^{(\beta)} \cdot \boldsymbol{n}=0 \quad \forall \beta \quad \text { on } S_{t}^{\text {dis }}
$$

3.1.3. Constitutive equations (including the latent hardening strain gradient extension). The Cauchy stress reads

$$
\sigma=\mathbb{L}\left(\varepsilon-\varepsilon^{p}\right)
$$

in which $\mathbb{L}$ is the elastic stiffness, henceforth assumed to be isotropic. The defect stress ensues from a power-law defect energy (already exploited in [39] in the context of phenomenological strain gradient plasticity):

$$
d=\mu \ell^{2}(\ell|\boldsymbol{\alpha}|)^{M-1} \boldsymbol{\alpha},
$$

where $\ell$ is an energetic length scale. $\sigma$ and $d$ are all the stresses coming from the free energy. In order to satisfy the 2 nd law, the remaining (dissipative) stresses must be such that

$$
\sum_{\beta=1}^{A}\left(s^{(\beta)} \dot{\gamma}^{(\beta)}+\omega_{\perp}^{(\beta)} \dot{\rho}_{\perp}^{(\beta)}+\omega_{\odot}^{(\beta)} \dot{\rho}_{\odot}^{(\beta)}+\omega_{N}^{(\beta)} \dot{\rho}_{N}^{(\beta)}\right)>0 \quad \forall\left(\underline{\dot{\gamma}}, \underline{\dot{\rho}}_{\perp}, \underline{\dot{\rho}}_{\odot}, \underline{\dot{\rho}}_{N}\right) \neq(\underline{0}, \underline{0}, \underline{0}, \underline{0}) .
$$


The choice presented next (see, e.g., [6,9]), extending the standard framework of crystal viscoplasticity, respects condition (3.7). Let us first define

$$
s^{(\beta)}=g^{(\beta)}(\lambda)\left(\frac{\dot{\gamma}_{\text {eff }}^{(\beta)}}{\dot{\gamma}_{0}}\right)^{N-1} \frac{\dot{\gamma}^{(\beta)}}{\dot{\gamma}_{0}},
$$

where $^{9}$

$$
\dot{\gamma}_{\text {eff }}^{(\beta)}=\sqrt{\left(\dot{\gamma}^{(\beta)}\right)^{2}+\left(L_{\perp} \dot{\rho}_{\perp}^{(\beta)}\right)^{2}+\left(L_{\odot} \dot{\rho}_{\odot}^{(\beta)}\right)^{2}+\left(L_{N} \dot{\rho}_{N}^{(\beta)}\right)^{2}} \quad \forall \beta
$$

involves three positive dissipative length scales $\left(L_{\perp}, L_{\odot}, L_{N}\right), \dot{\gamma}_{0}$ is a positive reference rate, $N>0$ is a rate sensitivity parameter, and $g^{(\beta)}(\lambda)$ is the resistance to dislocation glide, for which we put forward a strain gradient dependence, as follows.

Slip gradient dependent latent hardening. The resistance to dislocation glide $g^{(\beta)}(\lambda)$ is here given by the following extension of the latent hardening proposed in Peirce et al [11], in which the gradient dependence enters through both $\underline{\dot{\gamma}}_{\text {eff }}$, which is quite natural, and the term highlighted by the horizontal brace, involving the new gradient-dependent variable $\underline{\dot{\alpha}}$, defined below:

$$
\begin{aligned}
& \dot{g}^{(\beta)}(\lambda)=\mathcal{H}(\lambda) \sum_{\kappa=1}^{A}\left\{q \dot{\gamma}_{\text {eff }}^{(\kappa)}+[(1-q) \dot{\gamma}_{\text {eff }}^{(\kappa)}+\underbrace{\left(\frac{\ell \dot{\alpha}^{(\kappa)}}{\dot{\epsilon}_{0}}\right)^{n_{p}} \dot{\epsilon}_{0}}_{\text {grad. extension }}] \delta_{\beta \kappa}\right\} \\
&=\mathcal{H}(\lambda)\{\underbrace{\left(\frac{\ell \dot{\alpha}^{(\beta)}}{\dot{\epsilon}_{0}}\right)^{n_{p}} \dot{\epsilon}_{0}}_{\text {grad. extension }}+\sum_{\kappa=1}^{A}\left[q+(1-q) \delta_{\beta \kappa}\right] \dot{\gamma}_{\text {eff }}^{(\kappa)}\}, \quad g^{(\beta)}(\lambda=0)=\tau_{0}, \\
& \dot{\alpha}^{(\beta)}=\left|\sum_{\substack{\kappa=1 \\
\kappa \neq \beta}}^{A} N_{i m j}^{(\kappa)} \dot{\gamma}_{, m}^{(\kappa)}\right| \equiv\left|\sum_{\kappa=1}^{A}\left(1-\delta_{\kappa \beta}\right) N_{i m j}^{(\kappa)} \dot{\gamma}_{, m}^{(\kappa)}\right|, \quad \dot{\lambda}=\sum_{\beta=1}^{A}\left(\dot{\gamma}_{\text {eff }}^{(\beta)}+\ell \dot{\alpha}^{(\beta)}\right) .
\end{aligned}
$$

In the above equations (3.9)-(3.10), $\mathcal{H}(\lambda)$ is the self-hardening function, $\tau_{0}$ is a positive reference stress, $\delta_{\beta \kappa}$ is the Kronecker delta, $q$ is the conventional latent hardening parameter (typically, $q \in[1.2,1.4]$ in standard crystal plasticity [11]), and $n_{p}$ and $\dot{\epsilon}_{0}$ are further (new) material parameters governing the gradient extension of the latent hardening proposed here ${ }^{10}$. The self-hardening $\mathcal{H}(\lambda)$ is here conventionally defined as

$$
\mathcal{H}(\lambda)=h_{0} \operatorname{sech}^{2} \frac{h_{0} \lambda}{\tau_{s}-\tau_{0}},
$$

with $h_{0}$ a positive hardening rate and $\tau_{s}>\tau_{0}$ the saturation stress [11].

9 A more general expression for the effective slip rate is

$$
\dot{\gamma}_{\text {eff }}^{(\beta)}=\left(\left|\dot{\gamma}^{(\beta)}\right|^{p}+\left|L_{\perp} \dot{\rho}_{\perp}^{(\beta)}\right|^{p}+\left|L_{\odot} \dot{\rho}_{\odot}^{(\beta)}\right|^{p}+\left|L_{N} \dot{\rho}_{N}^{(\beta)}\right|^{p}\right)^{1 / p} \quad \forall \beta .
$$

Here, we choose $p=2$. However, $p \rightarrow \infty$, which should mostly lead to a larger strengthening prediction [7], has been proposed for phenomenological plasticity by Evans and Hutchinson [47] on the basis of the comparison with experimental results, and within this context, has been shown by Reddy [48] to correspond to a Tresca-like criterion on the maximum magnitude of the microstresses. As for most of the material parameters involved in the dissipative part of the constitutive prescriptions proposed here, even more dissipative length scales might be involved in the modelling by associating different lengths $L_{\mathrm{I}}^{(\beta)}$ to different systems $\beta$.

${ }^{10}$ Note that one of the three material parameters involved in the latent hardening gradient extension is redundant. Hence, we have chosen to take as material length scale the same parameter $\ell$ involved into definition (3.6) of the defect energy. 
The latent hardening strain gradient extension proposed here accounts, on a given slip system $\beta$, for the dislocation densities developed on the other slip systems (i.e. different from $\beta$ ) through $\dot{\alpha}^{(\beta)}$. In the presence of slip gradients, this gives a significant contribution to the latent hardening even when the plastic slips are very small, and this allows the qualitative description of what happens at the boundary layers, where dislocations pile up. This further source of higher-order coupling between slips, with respect to that provided by the defect energy (3.6) defined as a function of Nye's tensor, seems to be necessary to represent the DD results [49]. Moreover, in our investigation we have found that there is the need, in the SGCP modelling, of an irreversible contribution to the slip resistance $\dot{g}^{(\beta)}$ as that provided by $\dot{\alpha}^{(\beta)}$, incrementally related to GNDs. This vaguely agrees with the modelling of Gurtin and Ohno [26], who have developed a higher-order work-conjugate SGCP theory in which, in fact, the primal variable governing the defect energy consists of the integral along the loading history of the modulus of the dislocation density rate. Note that the model of Gurtin and Ohno [26], like that studied here, has the purpose of describing the interactions among slip systems.

The dissipative higher-order stresses are defined as

$$
\omega_{I}^{(\beta)}=\tau_{0} L_{I}^{2}\left(\frac{\dot{\gamma}_{\text {eff }}^{(\beta)}}{\dot{\gamma}_{0}}\right)^{N-1} \frac{\dot{\rho}_{I}^{(\beta)}}{\dot{\gamma}_{0}} \quad \text { with } I=\perp, \odot, N .
$$

With reference to this constitutive choice for the higher-order dissipative terms, let us remark that the substitution of the constant reference stress $\tau_{0}$ with $g^{(\beta)}(\lambda)$, in order to have a constitutive prescription closer to equation (3.8) for $s^{(\beta)}$, would not make any substantial quantitative difference for the present study because the representation of the DD results requires us to set $L_{I} \ll \ell, \forall I=\perp, \odot, N$.

3.1.4. The flow rule in terms of the plastic slips. For the sake of completeness, we rewrite the higher-order balance equation (3.3) in terms of displacement and plastic slips, through the relevant kinematic and constitutive equations provided above:

$$
\begin{gathered}
\underbrace{g^{(\beta)}\left(\frac{\dot{\gamma}_{\text {eff }}^{(\beta)}}{\dot{\gamma}_{0}}\right)^{N-1} \frac{\dot{\gamma}^{(\beta)}}{\dot{\gamma}_{0}}-\tau_{0} D_{i j}^{(\beta)}\left[\left(\frac{\dot{\gamma}_{\text {eff }}^{(\beta)}}{\dot{\gamma}_{0}}\right)^{N-1} \frac{\dot{\gamma}_{, j}^{(\beta)}}{\dot{\gamma}_{0}}\right]_{, i}}_{\text {dissipative hardening }}+2 \mu \mu_{i j}^{(\beta)} \sum_{\kappa=1}^{A} \gamma^{(\kappa)} \mu_{i j}^{(\kappa)} \\
\underbrace{-\mu \ell^{2} N_{k i l}^{(\beta)}\left[\left(\ell^{2} \sum_{\kappa=1}^{A} \gamma_{, m}^{(\kappa)} N_{s m t}^{(\kappa)} \sum_{\omega=1}^{A} \gamma_{, n}^{(\omega)} N_{s n t}^{(\omega)}\right)^{\frac{M-1}{2}} \sum_{\delta=1}^{A} \gamma_{, p}^{(\delta)} N_{k p l}^{(\delta)}\right]_{, i}}_{\text {energetic backstress }} \\
=2 \mu \mu_{i j}^{(\beta)} u_{i, j} \quad \beta=1, \ldots, A
\end{gathered}
$$

where

$$
\boldsymbol{D}^{(\beta)}=L_{\perp}^{2} \boldsymbol{m}^{(\beta)} \otimes \boldsymbol{m}^{(\beta)}+L_{\odot}^{2} \boldsymbol{t}^{(\beta)} \otimes \boldsymbol{t}^{(\beta)}+L_{N}^{2} \boldsymbol{n}^{(\beta)} \otimes \boldsymbol{n}^{(\beta)} .
$$

Equation (3.12) assumes, in the SGCP theory, the role of the flow rule.

\subsection{Simple shear of a constrained strip with multiple symmetric double slips}

In the boundary value problem described in section 1, within the context of crystal plasticity and contrary to the DD analyses, all the fields are independent of both $x_{1}$ and $x_{3}$, and, for each couple $\beta$ of slip systems, the two slips are equal and we call both of them $\gamma^{(\beta)}$. The sole 
non-vanishing components of the plastic strain and Nye's tensor are, respectively:

$$
\varepsilon_{12}^{p}=\sum_{\beta=1}^{B} \gamma^{(\beta)} \cos \left(2 \theta_{\beta}\right) \quad \alpha_{23}=2 \sum_{\beta=1}^{B}\left(\gamma^{(\beta)}\right)^{\prime} \sin ^{2} \theta_{\beta},
$$

where the symbol ' denotes a partial derivative computed with respect to $x_{2}$ and $\alpha_{23}$ is the density of the Burgers vector component in the $x_{2}$-direction due to geometrically necessary edge dislocations lying along $x_{3}$.

The conventional balance equation states that both $\sigma_{12}$ and $\sigma_{22}$ must be spatially uniform. Then, the boundary conditions (1.2) imply that $u_{2}$ (and $\sigma_{22}$ ) must be null everywhere, so that the problem is essentially unidimensional and henceforth we will simplify the notation by dropping some indices, as follows:

$x \equiv x_{2} \quad \varepsilon^{p} \equiv \varepsilon_{12}^{p} \quad \alpha \equiv \alpha_{23} \quad u \equiv u_{1} \quad \sigma \equiv \sigma_{12} \quad d \equiv d_{23}$

The sole non-trivial component of the standard constitutive law reads

$$
u^{\prime}-2 \sum_{\beta=1}^{B} \gamma^{(\beta)} \cos \left(2 \theta_{\beta}\right)=\frac{\sigma}{\mu}
$$

while the higher-order balance equation (3.12) (i.e. the unconventional flow rule) becomes

$$
\begin{aligned}
& \underbrace{g^{(\beta)}\left(\frac{\dot{\gamma}_{\text {eff }}^{(\beta)}}{\dot{\gamma}_{0}}\right)^{N-1} \frac{\dot{\gamma}^{(\beta)}}{\dot{\gamma}_{0}}-L_{\beta}^{2} \tau_{0}\left[\left(\frac{\dot{\gamma}_{\text {eff }}^{(\beta)}}{\dot{\gamma}_{0}}\right)^{N-1} \frac{\dot{\gamma}^{(\beta)^{\prime}}}{\dot{\gamma}_{0}}\right]^{\prime}}_{\text {dissipative hardening }}+2 \mu \cos \left(2 \theta_{\beta}\right) \sum_{\kappa=1}^{B} \gamma^{(\kappa)} \cos \left(2 \theta_{\kappa}\right) \\
& \underbrace{-2 \mu \ell^{2} \sin ^{2} \theta_{\beta}\left[\left(2 \ell\left|\sum_{\kappa=1}^{B} \gamma^{(\kappa)^{\prime}} \sin ^{2} \theta_{\kappa}\right|\right)^{M-1} \sum_{\omega=1}^{B} \gamma^{(\omega)^{\prime}} \sin ^{2} \theta_{\omega}\right]^{\prime}}_{\text {energetic backstress }}=\mu \cos \left(2 \theta_{\beta}\right) u^{\prime} \quad \forall \beta,
\end{aligned}
$$

where $L_{\beta}$ collects the two dissipative length scales, $L_{\perp}$ and $L_{N}$, relevant to this problem:

$$
L_{\beta}=\sqrt{\left(L_{\perp} \sin \theta_{\beta}\right)^{2}+\left(L_{N} \cos \theta_{\beta}\right)^{2}}
$$

and the definition of effective slip rate which turns out to be

$$
\dot{\gamma}_{\text {eff }}^{(\beta)}=\sqrt{\left(\dot{\gamma}^{(\beta)}\right)^{2}+\left[L_{\beta}\left(\dot{\gamma}^{(\beta)}\right)^{\prime}\right]^{2}} .
$$

About the latent hardening, equations (3.9)-(3.11) hold with

$$
\begin{aligned}
& \dot{g}^{(\beta)}=\mathcal{H}(\lambda) \sum_{\kappa=1}^{B}\left\{q \dot{\gamma}_{\mathrm{eff}}^{(\kappa)}+\left[(1-q) \dot{\gamma}_{\mathrm{eff}}^{(\kappa)}+\left(\frac{\ell \dot{\alpha}^{(\kappa)}}{\dot{\epsilon}_{0}}\right)^{n_{p}} \dot{\epsilon}_{0}\right] \delta_{\beta \kappa}\right\}, \\
& \dot{\alpha}^{(\beta)}=\left|2 \sum_{\kappa=1}^{B}\left(1-\delta_{\kappa \beta}\right)\left(\dot{\gamma}^{(\kappa)}\right)^{\prime} \sin ^{2} \theta_{\kappa}\right|, \quad \dot{\lambda}=\sum_{\beta=1}^{B}\left(2 \dot{\gamma}_{\mathrm{eff}}^{(\beta)}+\ell \dot{\alpha}^{(\beta)}\right)
\end{aligned}
$$

in which the particularization of equation (3.9) (first equation) into equation (3.14) for the symmetric simple shear has been accomplished just by substituting $A$ with $B$. This means that we do not account for the interaction between any two systems within the same couple, where, in fact, glide starts simultaneously in each system. 
The higher-order boundary conditions, essential to impose that dislocations are blocked when they reach the strip edges, in the case in which $L_{\perp}>0$ or $L_{N}>0$ are

$$
\gamma^{(\beta)}\left(x_{2}=0\right)=0 ; \quad \gamma^{(\beta)}\left(x_{2}=H\right)=0 \quad \forall \beta=1, \ldots, B .
$$

Actually, in the FE implementation presented below in section 4 we exploit the symmetries/antisymmetries of the problem and model only half of the strip region, with $x \in$ $[0, H / 2]$ and the boundary conditions (first equations in (1.1), (1.2), (3.16)), supplemented by

$$
\begin{aligned}
& u_{1}\left(x_{2}=H / 2\right)=\Gamma H / 2, \\
& u_{2}\left(x_{2}=H / 2\right)=0, \\
& \xi_{2}^{(\beta)}\left(x_{2}=H / 2\right)=0 \quad \forall \beta=1, \ldots, B,
\end{aligned}
$$

the last boundary condition being a static higher-order condition of the type (3.5) (second equation).

Finally, we note that the SGCP model proposed here, particularized to the simple shear problem and in the case of one sole couple of active systems $(B=1)$ without any conventional hardening (i.e. $g^{(\beta)}=\tau_{0} \forall \beta$ ), is totally analogous to the uniaxial strain gradient plasticity model studied by Anand et al [50]. Anand et al [50] implemented their model into an FE implicit algorithm and pointed out some numerical problems concerned with the implicit implementation. In the next section we report how we have addressed the analogous numerical issues we have encountered.

\section{FE implementation of the SGCP model for the simple shear problem}

\subsection{Weak form of the balance equations}

The starting point for developing the FE algorithm is the Principle of Virtual Work, which can be split into two equations as it must hold for any independent variation of the primal (kinematic) variables $u$ and $\gamma$, which constitute the $B+1$ nodal degrees of freedom:

$$
\begin{aligned}
& \int_{0}^{H_{\mathrm{el}}} \sigma \delta u^{\prime} \mathrm{d} x=0, \\
& 2 \int_{0}^{H_{\mathrm{el}}}\left\{\left[s^{(\beta)}-\sigma \cos \left(2 \theta_{\beta}\right)\right] \delta \gamma^{(\beta)}+\left(d \sin ^{2} \theta_{\beta}+\omega^{(\beta)}\right) \delta\left(\gamma^{(\beta)}\right)^{\prime}\right\} \mathrm{d} x=0 \quad \forall \beta,
\end{aligned}
$$

where $\omega^{(\beta)}$ is the combination of $\omega_{\perp}^{(\beta)}$ and $\omega_{N}^{(\beta)}$ which turns out to be work-conjugate with $\gamma^{(\beta)^{\prime}}$ (see, e.g., [7]) and $H_{\mathrm{el}}$ is the finite element length.

4.1.1. FE approximation. We employ two-noded isoparametric linear elements, both for the displacement $u$ and the slips $\gamma$. The facts that the kinematic relation between $u$ and $\gamma$ is enforced in a weak form and that only first-order derivatives of $u$ and $\underline{\gamma}$ appear in the equations to be solved, allows the use of $C^{0}$-shape functions both for the displacement and the plastic slips $^{12}$. Hence, the balance equations (4.1)-(4.2) can be rewritten in the intrinsic coordinate $r$,

${ }^{11}$ Otherwise, for the case of no dissipative higher-order terms (i.e. $L_{\perp}=L_{N}=0$ ), see $[5,46]$.

${ }^{12}$ Also, Evers et al [21] employed $C^{0}$-continuous finite elements in order to implement their backstress-based SGCP theory. A study on the best plane strain finite element formulation for higher-order SGCP, within the large deformation regime, has been carried out by Kuroda [35] (see also the references therein). 
which takes values equal to -1 at the node $A$ and 1 at the node $B$ in the parent element:

$$
\begin{aligned}
& \int_{-1}^{1} \sigma \frac{1}{2}\left(\delta u_{B}-\delta u_{A}\right) \mathrm{d} r=0, \\
& \int_{-1}^{1}\left\{\{\left[s^{(\beta)}-\sigma \cos \left(2 \theta_{\beta}\right)\right] \underbrace{\frac{1-r}{2}}_{N_{A}^{(r)}} H_{\mathrm{el}}-\left(\mathrm{d} \sin ^{2} \theta_{\beta}+\omega^{(\beta)}\right)\} \delta \gamma_{A}^{(\beta)}\right. \\
& \left.+\{\left[s^{(\beta)}-\sigma \cos \left(2 \theta_{\beta}\right)\right] \underbrace{\frac{1+r}{2}}_{N_{B}(r)} H_{\mathrm{el}}+\left(\mathrm{d} \sin ^{2} \theta_{\beta}+\omega^{(\beta)}\right)\} \delta \gamma_{B}^{(\beta)}\right\} \mathrm{d} r=0 \quad \forall \beta
\end{aligned}
$$

where $u_{I}, \gamma_{I}^{(\beta)}$ and $N_{I}(r)$, with $I=A, B$, are the nodal values of displacement and plastic slips and the interpolation functions, respectively.

4.1.2. Numerical time integration. We indicate with $\zeta_{t}$ and $\Delta \zeta$ the value of the variable $\zeta$ at the beginning of the time step and its increment within the step, respectively. By accounting for the chosen FE interpolation, we have:

$$
\begin{aligned}
& \zeta_{t}=\zeta_{t A} \frac{1-r}{2}+\zeta_{t B} \frac{1+r}{2}, \\
& \Delta \zeta=\Delta \zeta_{A} \frac{1-r}{2}+\Delta \zeta_{B} \frac{1+r}{2}
\end{aligned}
$$

in terms of the nodal values $\zeta_{t A}, \zeta_{t B}, \Delta \zeta_{A}$ and $\Delta \zeta_{B}$.

Hence, we adopt the generalized trapesodial integration scheme, in which the relevant quantities are evaluated at

$$
\zeta_{t}+v \Delta \zeta
$$

with $v \in[0,1]$ ( $v=1$ corresponding to the Backward-Euler scheme). By defining the rate within the time step $\Delta t$ as

$$
\dot{\zeta}=\frac{\Delta \zeta}{\Delta t},
$$

we obtain the following expressions for the stresses:

$$
\begin{aligned}
& \sigma=\sigma_{t}+v \mu\left(\frac{\Delta u_{B}-\Delta u_{A}}{H_{\mathrm{el}}}-2 \sum_{\beta=1}^{B} \Delta \gamma^{(\beta)} \cos \left(2 \theta_{\beta}\right)\right) \\
& s^{(\beta)}=\frac{g_{t}^{(\beta)}+v \Delta g^{(\beta)}}{\left(\Delta t \dot{\gamma}_{0}\right)^{N}}\left[\left(\Delta \gamma^{(\beta)}\right)^{2}+\left(L_{\beta} \frac{\Delta \gamma_{B}^{(\beta)}-\Delta \gamma_{A}^{(\beta)}}{H_{\mathrm{el}}}\right)^{2}\right]^{\frac{N-1}{2}} \Delta \gamma^{(\beta)} \quad \forall \beta, \\
& d=2 \mu \ell^{2}\left\{2 \ell\left|\sum_{\beta=1}^{B}\left(\frac{\gamma_{t B}^{(\beta)}-\gamma_{t A}^{(\beta)}}{H_{\mathrm{el}}}+v \frac{\Delta \gamma_{B}^{(\beta)}-\Delta \gamma_{A}^{(\beta)}}{H_{\mathrm{el}}}\right) \sin ^{2} \theta_{\beta}\right|\right\}^{M-1} \\
& \times \sum_{\beta=1}^{B}\left(\frac{\gamma_{t B}^{(\beta)}-\gamma_{t A}^{(\beta)}}{H_{\mathrm{el}}}+v \frac{\Delta \gamma_{B}^{(\beta)}-\Delta \gamma_{A}^{(\beta)}}{H_{\mathrm{el}}}\right) \sin ^{2} \theta_{\beta}, \\
& \omega^{(\beta)}=\frac{L_{\beta}^{2} \tau_{0}}{\left(\Delta t \dot{\gamma}_{0}\right)^{N}}\left[\left(\Delta \gamma^{(\beta)}\right)^{2}+\left(L_{\beta} \frac{\Delta \gamma_{B}^{(\beta)}-\Delta \gamma_{A}^{(\beta)}}{H_{\mathrm{el}}}\right)^{2}\right]^{\frac{N-1}{2}} \frac{\Delta \gamma_{B}^{(\beta)}-\Delta \gamma_{A}^{(\beta)}}{H_{\mathrm{el}}} \quad \forall \beta,
\end{aligned}
$$


where

$$
\begin{gathered}
\Delta g^{(\beta)}=\underbrace{h_{0} \operatorname{sech}^{2} \frac{h_{0}\left(\lambda_{t}+v \Delta \lambda\right)}{\tau_{s}-\tau_{0}}}_{\mathcal{H}(\lambda)}\left\{\left(\dot{\epsilon}_{0} \Delta t\right)^{1-n_{p}}[\ell \underbrace{\ell 2 \sum_{\kappa=1}^{B}\left(1-\delta_{\kappa \beta}\right) \frac{\Delta \gamma_{B}^{(\kappa)}-\Delta \gamma_{A}^{(\kappa)}}{H_{\mathrm{el}}} \sin ^{2} \theta_{\kappa} \mid}_{\Delta \alpha^{(\beta)}}]^{n_{p}}\right. \\
+\sum_{\kappa=1}^{B}\left[q+(1-q) \delta_{\beta \kappa}\right] \underbrace{\sqrt{\left(\Delta \gamma^{(\kappa)}\right)^{2}+\left(L_{\kappa} \frac{\Delta \gamma_{B}^{(\kappa)}-\Delta \gamma_{A}^{(\kappa)}}{H_{\mathrm{el}}}\right)^{2}}}_{\Delta \gamma_{\mathrm{efi}}^{(\kappa)}}\}
\end{gathered}
$$

and

$$
\begin{aligned}
\Delta \lambda= & \sum_{\beta=1}^{B}\left(2 \Delta \gamma_{\mathrm{eff}}^{(\beta)}+\ell \Delta \alpha^{(\beta)}\right)=2 \sum_{\beta=1}^{B}\left\{\sqrt{\left(\Delta \gamma^{(\beta)}\right)^{2}+\left(L_{\beta} \frac{\Delta \gamma_{B}^{(\beta)}-\Delta \gamma_{A}^{(\beta)}}{H_{\mathrm{el}}}\right)^{2}}\right. \\
& \left.+\ell\left|\sum_{\kappa=1}^{B}\left(1-\delta_{\kappa \beta}\right) \frac{\Delta \gamma_{B}^{(\kappa)}-\Delta \gamma_{A}^{(\kappa)}}{H_{\mathrm{el}}} \sin ^{2} \theta_{\kappa}\right|\right\} .
\end{aligned}
$$

The above expressions for the stresses have to be substituted into equations (4.3)-(4.4) to obtain the residual to be implemented in the uel. The residual is defined as the opposite of the left-hand sides of equations (4.3)-(4.4) differentiated with respect to the nodal degrees of freedom variations $\delta u_{A}, \delta \underline{\gamma}_{A}, \delta u_{B}$, and $\delta \underline{\gamma}_{B}$.

4.1.3. The consistent jacobian. The consistent jacobian to be implemented in the uel is defined as the opposite of the residual differentiated with respect to the nodal degrees of freedom increments $\Delta u_{A}, \Delta \underline{\gamma}_{A}, \Delta u_{B}$, and $\Delta \underline{\gamma}_{B}$. The element stiffness matrix $\underline{\underline{K}}$ has the dimension $2(B+1) \times 2(B+1)$ and the expressions for all its components are given in the appendix. Here, with reference to the appendix, we note that $\underline{\underline{K}}$ turns out to be unsymmetric, e.g., because of the integrals left unsolved in equation (6.2). Also, many numerical issues have to be addressed in order to obtain an efficient code. Among them, we remark that some contributions of the consistent jacobian, for the relevant range of the rate sensitivity parameter $N \in(0 ., 1$.$) , become numerically very large for a very small amount of plasticity (that is, for$ $\Delta \gamma_{\text {eff }}^{(\beta)} \rightarrow 0$ ). This unfavourable behaviour is emphasized as $N$ approaches zero (i.e. as the model tends to be rate independent).

Also, for a given the number of degrees of freedom, the FE model is more efficient if the mesh is finer where dislocations pile up (and slip gradients are maximum).

Our numerical tests showed that the most efficient time integration scheme is Backward Euler, obtained by setting $v=1$ in the generalized trapesoidal rule. The results presented in the following section refer to this choice.

\section{Comparison between DD and SGCP results}

The comparison between the results obtained from DD and SGCP is documented in figures 5-8, which also report, as reference, the prediction of the conventional crystal plasticity (obtainable in the limit of vanishing material length scales). The SGCP model is characterized by the following set of material parameters: $\mu \approx 26316 \mathrm{MPa}, \tau_{0}=12 \mathrm{MPa}, \tau_{s}=20 \mathrm{MPa}$, $h_{0}=500 \mathrm{MPa}, \dot{\gamma}_{0}=2 . \mathrm{E}-6 \mathrm{~s}^{-1}, q=0.6, n_{p}=0.15, \dot{\epsilon}_{0}=1 . \mathrm{E}-4 \mathrm{~s}^{-1}, N=0.165, M=1$, $\ell=0.6 \mu \mathrm{m}$ and $L_{\perp}=L_{\mathrm{N}}=0.12 \mu \mathrm{m}$. Note that $\dot{\gamma}_{0}$ and $\dot{\epsilon}_{0}$ scale linearly with the total time of the analysis, in this case equal to $1 . \mathrm{E}-5 \mathrm{~s}$. 


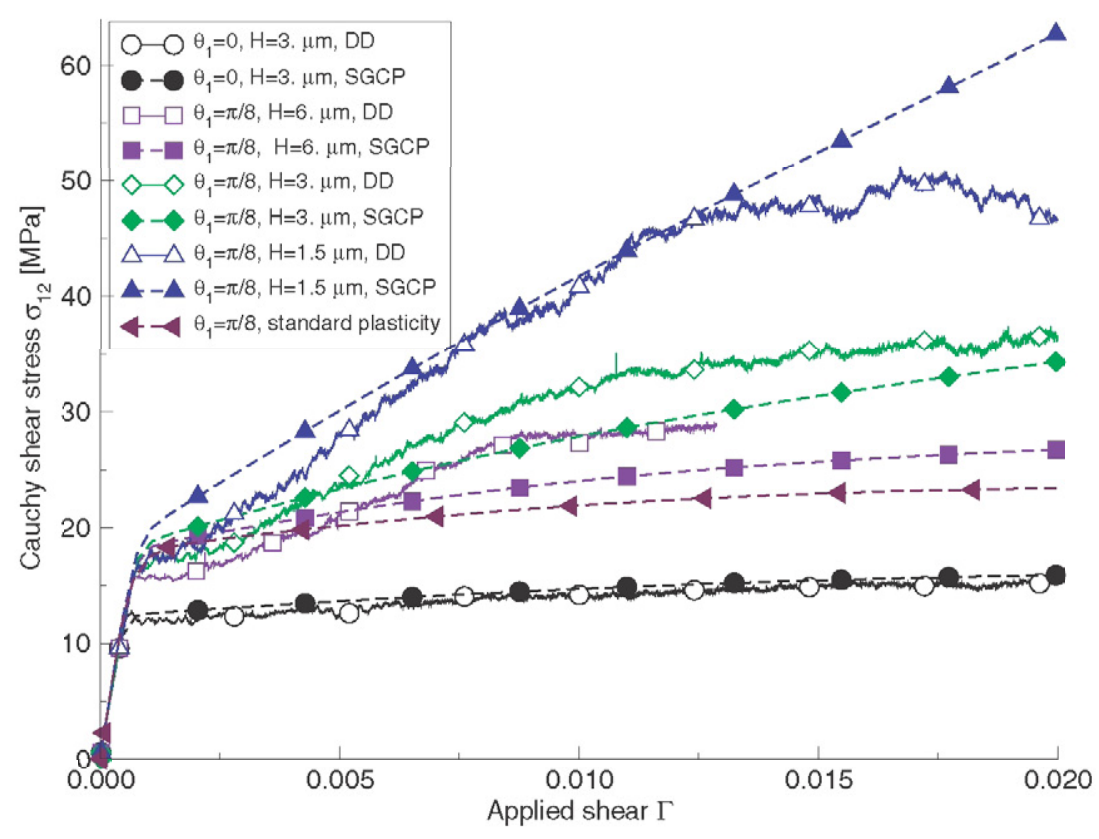

Figure 5. Comparison between DD and SGCP results on a constrained crystalline strip with a single couple of symmetric slip systems.

The quality of the numerical results obtained with the SGCP FE code has been checked by refining the mesh until stabilization of the results. The finest mesh employed consists of a 72000 element discretizing half strip. The element size decreases from the middle of the strip to the boundary by a ratio of 0.9999 between two consecutive elements, so that the element having a node on the boundary has length of about $7.5 \mathrm{E}-4$ the length of the element having one node in the strip middle plane.

The comparison given in figures 5-8 is overall quite satisfactory from the quantitative point of view if judged in terms of a global increase in hardening accompanied with diminishing size. In fact, note that the material parameters above have not been set by exploiting any specific identification method: they have been instead obtained by a lengthy trial and error procedure. Hence, we expect room for improvement in the match between DD and SGCP results. However, this is beyond the scope of this article, in which we mainly wish to point out the huge size effect on the latent hardening predicted by DD, which seems to require, to be described by higher-order work-conjugate SGCP theory, a strain gradient extension of the conventional latent hardening. Let us further note that, just after yielding, the difference between the DD and SGCP results is mainly due to two causes: (i) the SGCP here employed is not endowed with a softening law able to represent the sudden and simultaneous activation of several sources leading, in DD, to a stress decay after the first yielding; (ii) in order to reach convergence, the SGCP implicit algorithm has required the introduction, through appropriate choice of the material parameters, of an amount of rate dependence and higher-order dissipation, actually larger than that exhibited by DD, raising the stress-strain curve predicted by SGCP. The comparison between figures 5 and 6 shows that the SGCP model seems to be able to predict an interesting peculiarity of the DD response: the size effect is quite small for strips larger than about $H=3 \mu \mathrm{m}$ in the case of two couples of slip systems (suggesting a sort of exhaustion of the size effect for strips a bit larger), while this is not the case for the strip endowed with one sole couple of systems. 


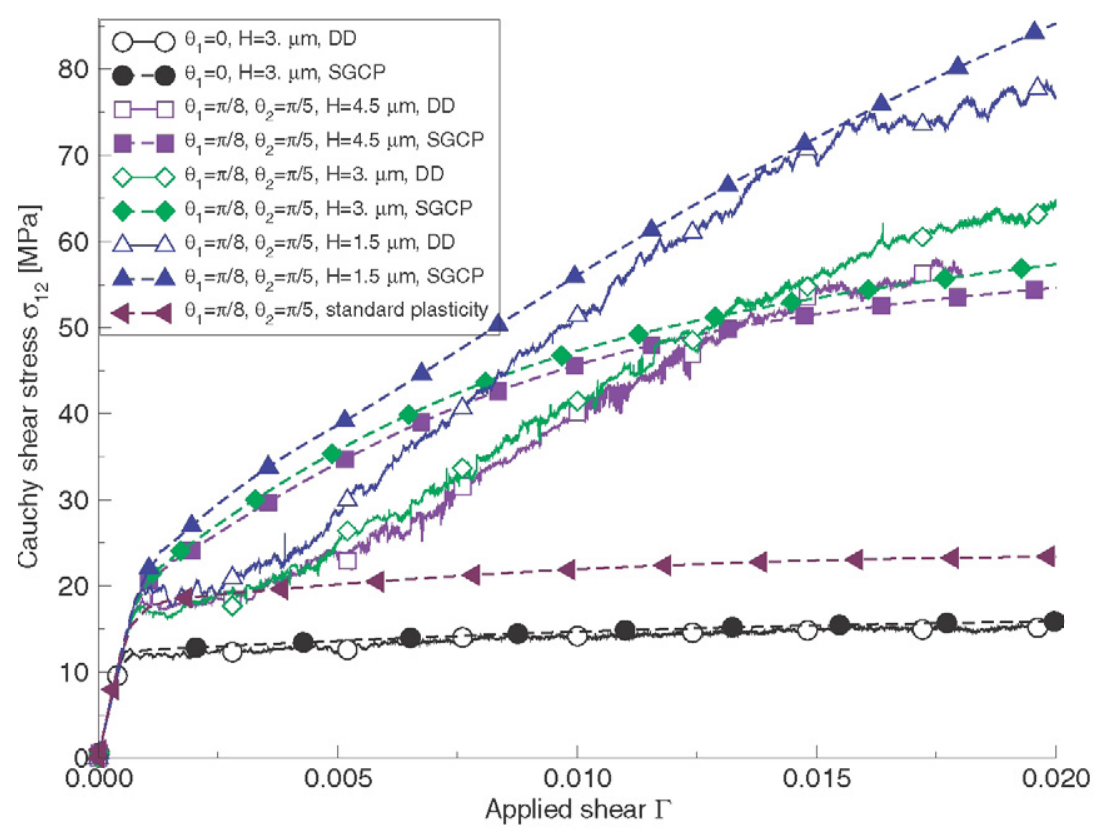

Figure 6. Comparison between DD and SGCP results on a constrained crystalline strip with two couples of symmetric slip systems.

The value of the energetic length scale $\ell=0.6 \mu \mathrm{m}$ has been set just by trying to reach the best match between DD and SGCP results. It is lower than the values identified by Nicola et al [29], ranging from 1.5 and $4.5 \mu \mathrm{m}$ depending on the chosen form of the defect energy ${ }^{13}$. However, as derived by Groma and coworkers (see, e.g., [52]), the material length should be related to the average Statistically Stored Dislocations spacing, which can be estimated by the square root of the strip area divided by the total number of dislocations, which, in our benchmark, reaches a plateau at about the end of DD analyses with $\Gamma=2 \%$; by taking the plateau value for the total number of dislocations, we obtain a lower bound for the length of about $\approx 0.1 \mu \mathrm{m}$, in fact smaller than that identified by comparing DD with SGCP. Of course, contrary to the dislocation density-based modelling developed in [52], our SGCP modelling does not account for the dependence of the material length scale on the dislocation density.

Note that, for what concerns the conventional self-hardening parameters, Peirce et al [11] suggest $h_{0}=8.9 \tau_{0}$ and $\tau_{s}=1.8 \tau_{0}$. Our identification based on the DD results for a strip with zero-inclined slip systems (see the black plots in figures 5 and 6 ) provides $\tau_{s} / \tau_{0} \approx 1.7$, while $h_{0} / \tau_{0} \approx 42$. About the value $q=0.6$ of the conventional latent hardening parameter, we note that a value larger than $\approx 1$ makes the plastic $\operatorname{sip} \gamma^{(2)}(x)$ numerically vanish. However, $q$ does not have a big influence on the predictions of the SGCP model, in which $\gamma^{(2)}(x)$ mostly affects the results through its gradient. In the standard crystal plasticity case (see the brown plots in figures 5-8), $q=0.6$ is large enough to make the stress-strain curves for $B=1$ and $B=2$ almost identical (that is, in the case $B=2, \gamma^{(2)}(x)$ is negligible with respect to $\gamma^{(1)}(x)$ ).

${ }^{13}$ Among the many possible sources of discrepancy in the material length scale as identified by Nicola et al [29] and by us, let us here mention the different choice of some parameters in the DD simulations: Nicola et al [29] chose (see, e.g., Nicola and van der Giessen [51]) source density $=60 \mu \mathrm{m}^{-2}$, obstacle strength $=150 \mathrm{MPa}$, and obstacle density $=60 \mu \mathrm{m}^{-2}$, while in this work those parameters are set to $42 \mu \mathrm{m}^{-2}, 300 \mathrm{MPa}$, and $126 \mu \mathrm{m}^{-2}$, respectively. 


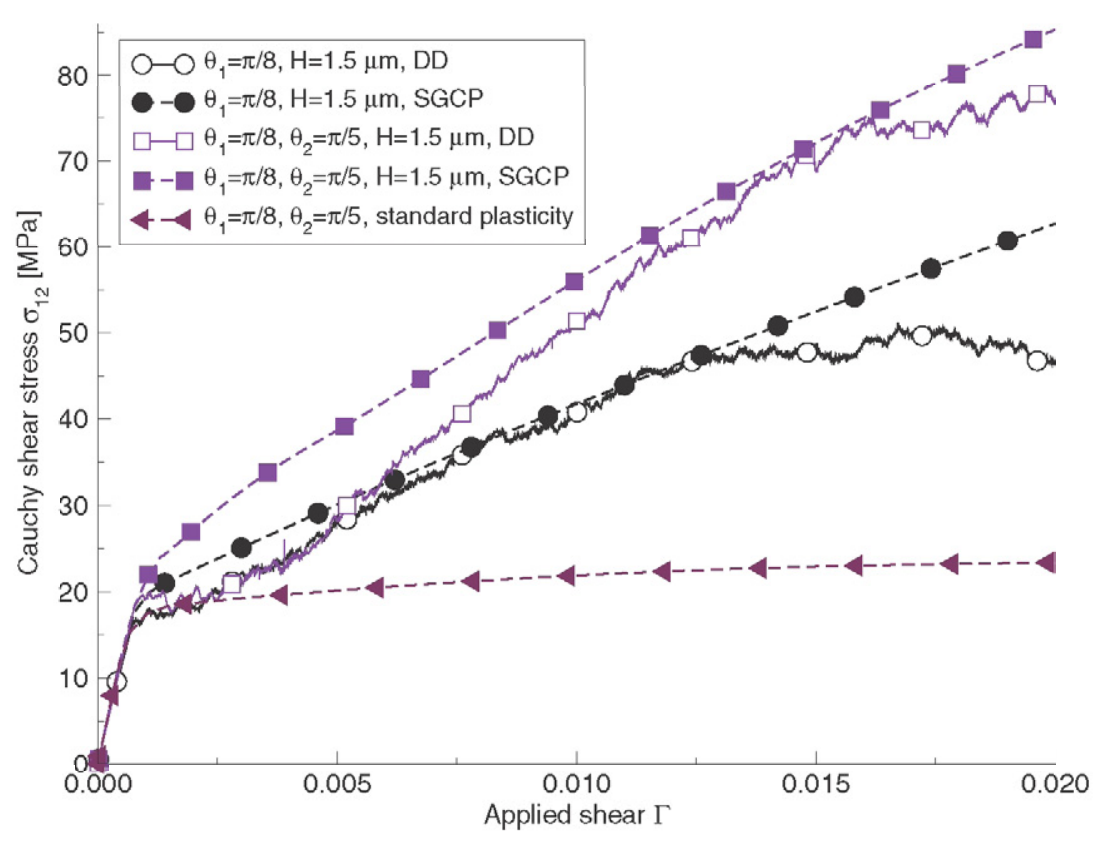

Figure 7. Comparison between DD and SGCP results on a constrained crystalline strip of size $H=1.5 \mu \mathrm{m}$ endowed with either one couple or two couples of symmetric slip systems.

Since DD results are quite rate insensitive, we set $N$ as small as possible, compatibly with the algorithm efficiency (decreasing $N$ makes convergence more difficult). Note that in order to represent the DD results with SGCP we found that smaller the parameter $N$ larger the parameter $\dot{\epsilon}_{0}$.

For the case $H=1.5 \mu \mathrm{m}$ with two couples of slip systems, figure 9 compares the SGCP predictions provided by the use of (i) the novel gradient dependent latent hardening, (ii) the standard latent hardening of Peirce et al [11] and (iii) no latent hardening at all. As reference, in figure 9 we have also plotted the stress-strain curves provided by standard crystal plasticity and by SGCP for the case of one sole couple of slip systems. The absence of the novel gradient dependent latent hardening leads to stress-strain curves which coincide with that for one sole couple of slip systems until the gradient term $\operatorname{div} \boldsymbol{\xi}^{(2)}$ becomes large enough to require a significant plastic slip $\gamma^{(2)}$ to satisfy the unconventional balance equation (3.3) for the second couple of slip systems. This is further documented by figure 10, where the plastic slips are plotted as functions of the applied shear for the SGCP model with conventional latent hardening. After the second couple becomes active, the difference between the two SGCP models with no latent hardening at all or standard latent hardening is small (see figure 9), the latter having latent hardening coefficient $(q=0.6)$ such that the activation of the second couple makes the response more compliant. This analysis shows the phenomenological reason why there is the need for a slip gradient dependent latent hardening, which allows a quite small plastic slip $\left(\gamma^{(2)}\right)$ to strongly influence the stress-strain response just after the first yielding. For the case $H=3 \mu \mathrm{m}$, a similar analysis shows that the stress-strain curves provided by the two SGCP models with no latent hardening at all or standard latent hardening coincide with the stress-strain curve for one sole couple of slip systems within the whole range $\Gamma \in[0,0.02]$; this is due to the fact that for this larger strip the gradient term $\operatorname{div} \boldsymbol{\xi}^{(2)}$ is less important so that the second couple of slip systems remains substantially inactive. 


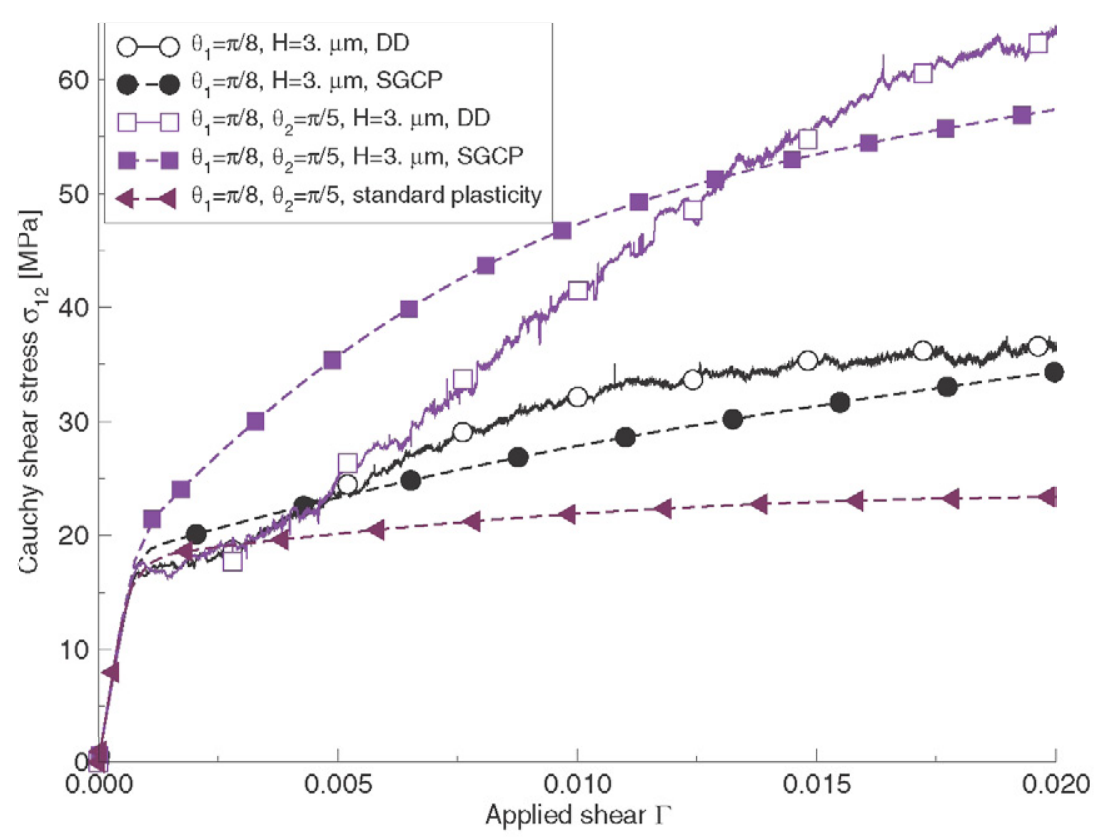

Figure 8. Comparison between DD and SGCP results on a constrained crystalline strip of size $H=3 \mu \mathrm{m}$ endowed with either one couple or two couples of symmetric slip systems.

In figures 11-15 we further analyze the results of the SGCP model. From figures 11 and 12 one easily obtains, through equation (3.13) (first equation), that the plastic strain diminishes with the strip height $H$. Regarding the negative value of $\gamma^{(2)}(x)$ shown in figures 10 and 12 , we note that it is due to the coupling of the higher-order balance equations (3.3)-(3.4) provided by the use of Nye's tensor as a primal higher-order variable governing the defect energy. Figure 13 demonstrates this by showing $\gamma^{(2)}(x)$ along the strip height for various combinations of the latent hardening terms implemented: the most negative $\gamma^{(2)}(x)$ is obtained assuming no dissipative latent hardening at all, where the coupling of the higher-order balance equations is provided by the defect stress only.

Also, our numerical results show that for values of the applied shear $\Gamma$ lower than about $0.01, \gamma^{(2)}(x)>0 \forall x$; then, there is an inversion in the evolution of $\gamma^{(2)}(x)$, which is not a monotonic function of the applied load, at least in most of the strip. This is due to the fact that, in most of the strip, $\gamma^{(1)^{\prime}}(x)$ is not a monotonic function of the applied load.

The material parameter $M$ governing the defect energy (3.6) has been set equal to 1 , so that the defect energy employed is quadratic, as formerly proposed by Gurtin [17]. With respect to previous studies of phenomenological strain gradient plasticity [39], our numerical tests have confirmed that as $M$ goes from 1 to 0 as the strengthening component of the energetic size effect increases, whereas strain hardening variation decreases. Hence, $M<1$ is inappropriate to describe the DD results we have obtained. We also found that, for the boundary value problem here concerned, for $M>1$, increasing $M$ makes the effect of the plastic slip gradient too weak. Of course, before proposing the unconventional latent hardening as in equations (3.9)(3.10), we attempted to describe the DD results by exploiting only the coupling provided by the defect energy and by the conventional latent hardening; we could not find any satisfactory combination of the relevant material parameters (basically, $M$ and $q$, for quite small $N$-see, e.g., Bardella and Segurado [49]): in that case, to appreciate some strain hardening increase 


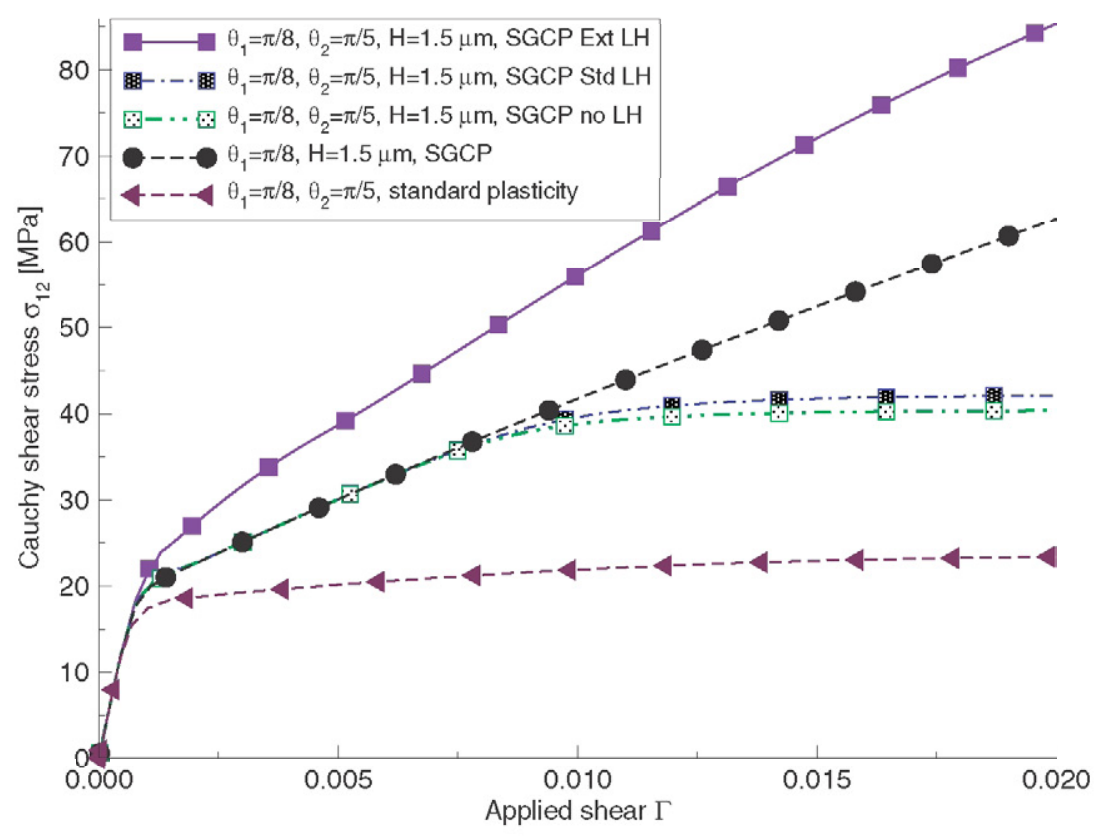

Figure 9. Comparison among SGCP results on a constrained crystalline strip of size $H=1.5 \mu \mathrm{m}$, endowed with either one couple or two couples of symmetric slip systems, for different latent hardening laws. 'Ext LH', 'Std LH', and 'no LH' refer to the novel strain gradient extended latent hardening, the conventional latent hardening of Perice et al [11], and the absence of latent hardening, respectively.

with diminishing size, we needed too large of a rate sensitivity parameter $N$ combined with an unrealistic value of $q \approx 30$.

Figures 14 shows the increase in the GND density accompanied with diminishing size for the case $B=2$, whereas figure 15 compares the GND density for the cases $B=1$ and $B=2$.

\section{Concluding remarks}

We have investigated the behaviour of a crystalline strip deforming under multislip, within the small strain regime, while sheared between two bodies impenetrable by dislocations. We have focused our attention on the effect of the strip size on the mechanical response; to this purpose we have varied the strip height within the range $[1.5 \mu \mathrm{m}, 6 \mu \mathrm{m}]$.

First, we have obtained pseudo-experimental results by means of discrete Dislocation Dynamics (DD) simulations, which have revealed a very conspicuous size effect on the latent hardening, consisting of an increase in the strain hardening accompanied with diminishing size. DD simulations predict almost no strengthening, that is, the stress recognized as the initial yield stress is unaffected by the strip size.

Second, we have developed a higher-order, work-conjugate Strain Gradient Crystal Plasticity (SGCP) theory with the purpose of discussing and describing the DD results. The SGCP model able to represent the DD results to some extent consists of a strain gradient extension of the standard crystal plasticity model of Peirce et al [11]. In particular, the SGCP model is based on the theories developed in [5-9], involves both energetic and dissipative unconventional terms, and, as a main novelty, puts forward a strain gradient extension of the standard latent hardening of Peirce et al [11]. 


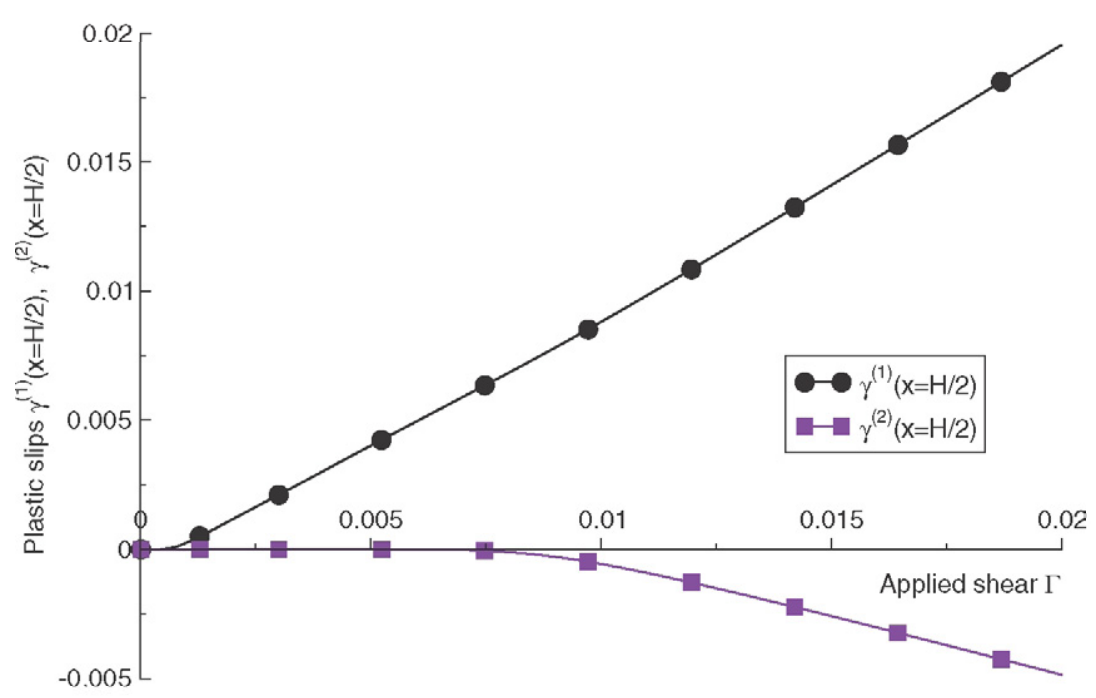

Figure 10. Plastic slips as functions of the applied shear $\Gamma$ predicted by the SGCP model with the conventional latent hardening of Perice et al [11] (no strain gradient extension), for a constrained crystalline strip of size $H=1.5 \mu \mathrm{m}$ endowed with two couples of symmetric slip systems.

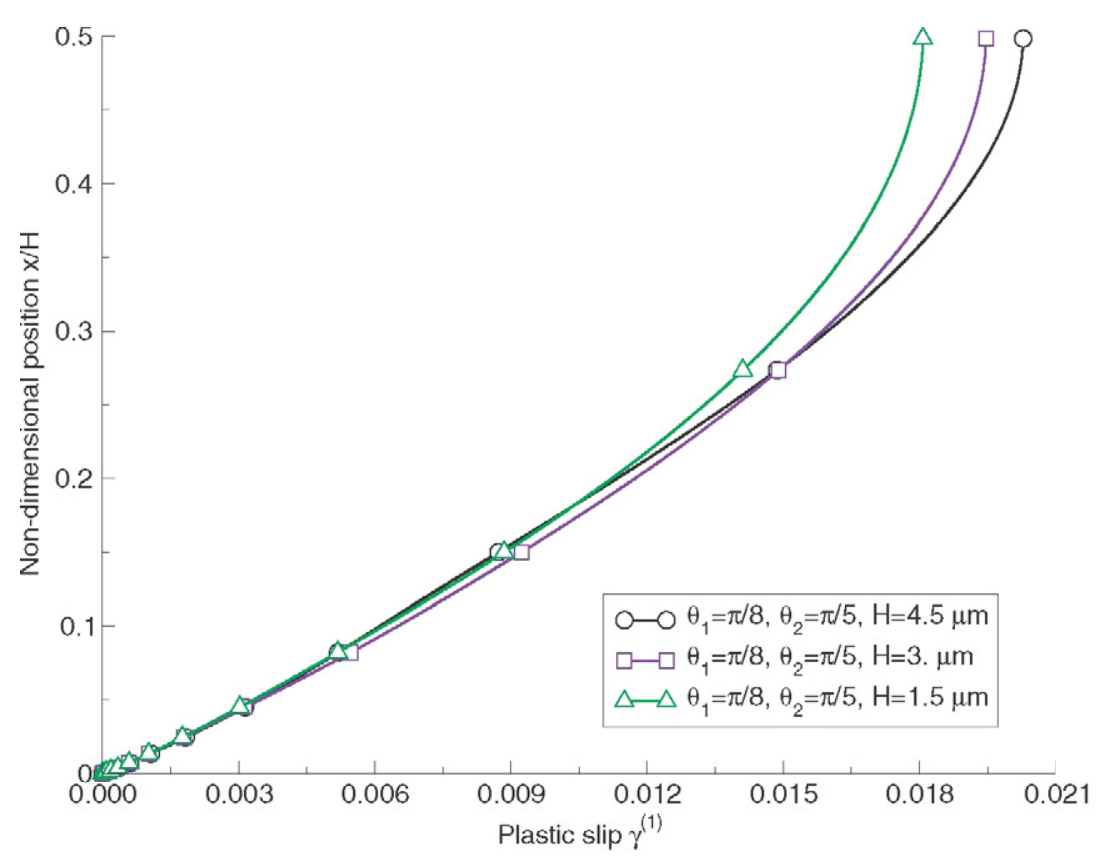

Figure 11. SGCP results in terms of plastic slip $\gamma^{(1)}$ on a constrained crystalline strip endowed with two couples of symmetric slip systems, with applied shear $\Gamma=0.02$.

Hence, our main conclusion, numerically proved by means of DD simulations, is that there exists a very large size effect influencing the latent hardening within the size range in which SGCP applies. In order to describe such a size effect by using SGCP we found the need to put forward a (further) strain gradient dependence involved in the latent hardening law. 


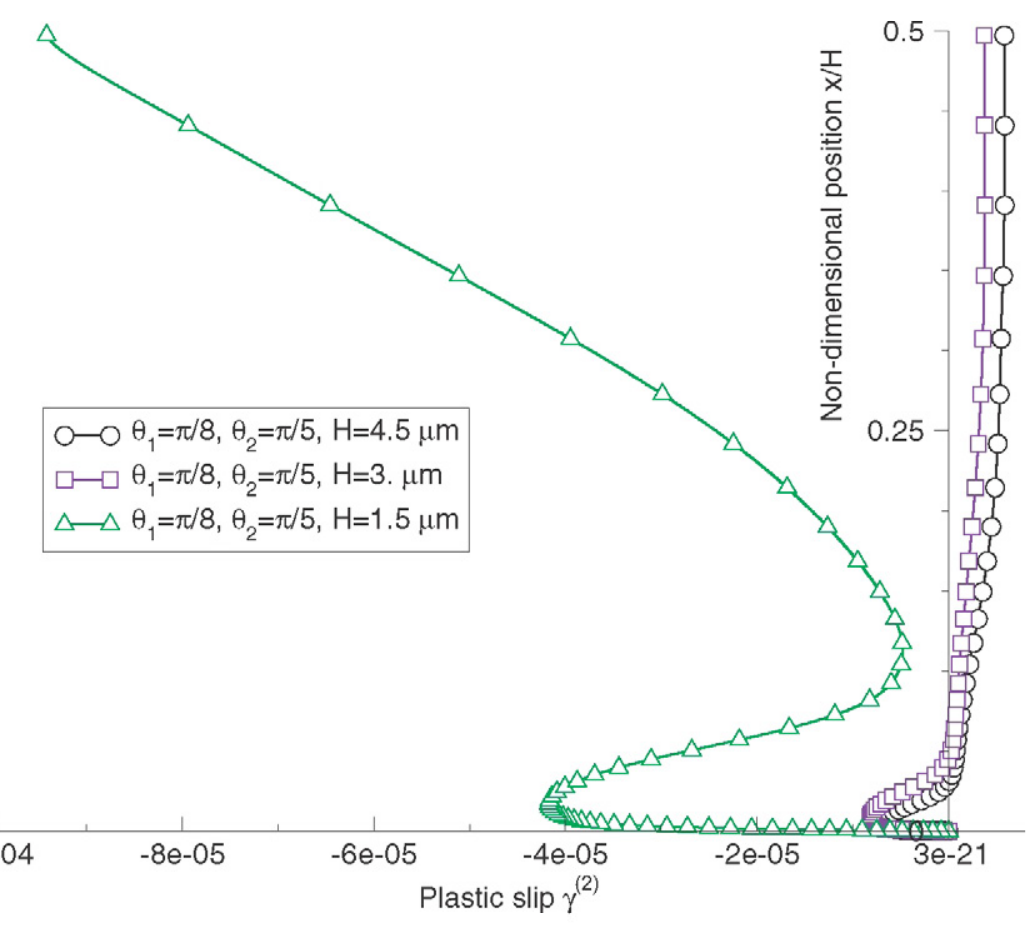

Figure 12. SGCP results in terms of plastic slip $\gamma^{(2)}$ on a constrained crystalline strip endowed with two couples of symmetric slip systems, with applied shear $\Gamma=0.02$.

Further DD analyses shall be designed in the future in order to obtain more pseudoexperimental results, on the basis of which it should be possible to obtain better insight on the most appropriate constitutive ingredients of the SGCP model, among those proposed in the literature (e.g., Gurtin and Ohno [26]) and our present contribution. For instance, the definition of a non-convex potential able to describe the dislocation patterning might be important; this might be obtained by either a non-convex conventional latent hardening (e.g., Ortiz and Repetto [53] as employed by Yalçinkaya et al [24]) or a non-convex defect energy, or both.

Even for the specific boundary value problem considered here there is still room for much work, for instance, by considering different relative orientations among slip systems. Investigating the cyclic behaviour would be very important and might allow the comparison between DD and SGCP in terms of plastic strain, a priori undefined in DD; this would also constitute an important test for the latent hardening strain gradient extension proposed here. Also, the implementation of a specific identification procedure for the material parameters of the SGCP model would be quite important.

\section{Acknowledgments}

Work financed by the Italian Ministry of Education, University, and Research (MIUR), by the Spanish Ministry of Science and Innovation (Project MAT2009-14396), and by the Integrated Action Italy-Spain titled 'Modelling the size effects in metals at the nano/microscale by combining strain gradient crystal plasticity and discrete dislocation dynamics' (Italian reference: IT090C205F, Spanish reference: HI2008-0147).

Professors Marc G D Geers, Christian F Niordson, Viggo Tvergaard, and István Groma are acknowledged for helpful discussions. 


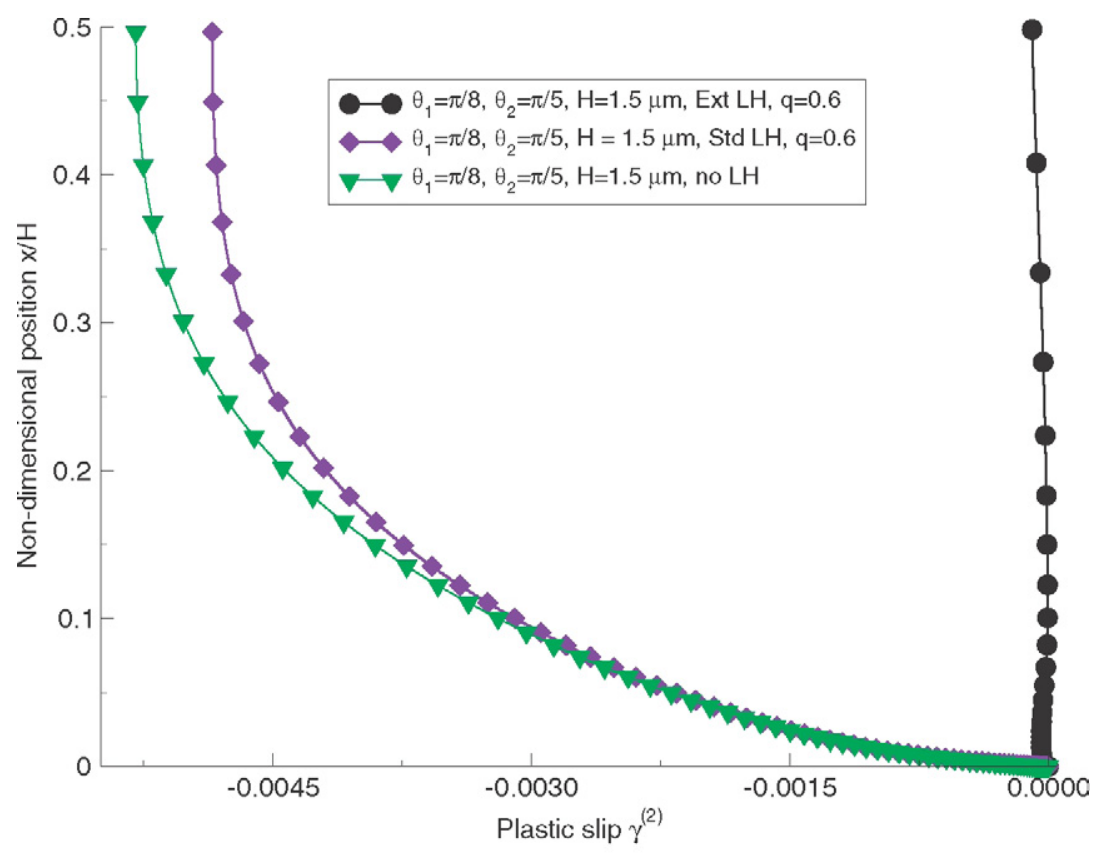

Figure 13. SGCP results in terms of plastic slip $\gamma^{(2)}$ on a constrained crystalline strip endowed with two couples of symmetric slip systems, with applied shear $\Gamma=0.02$ : effect of different latent hardening laws. 'Ext LH', 'Std LH', and 'no LH' refer to the novel strain gradient extended latent hardening, the conventional latent hardening of Perice et al [11], and the absence of latent hardening, respectively.

\section{Appendix. The consistent jacobian}

Here, we provide explicit expressions for the components of the consistent jacobian $\underline{K}$. Its components solely related to the displacement read

$K_{u_{I} u_{J}}=K_{u_{J} u_{I}}=\int_{-1}^{1}\left(\frac{\mathrm{d} N_{I}(r(x))}{\mathrm{d} x} \frac{\mathrm{d} N_{J}(r(x))}{\mathrm{d} x}\right) v \mu \frac{H_{\mathrm{el}}}{2} \mathrm{~d} r=(-1)^{1+\delta_{I J}} \frac{v \mu}{H_{\mathrm{el}}}$,

where, here and henceforth, the capital letters $I$ and $J$ may assume values $A$ or $B$ depending on the node considered, $\delta_{I J}=1$ if $I=J$ or $\delta_{I J}=0$ if $I \neq J$. The second equality in equation (6.1) follows from the chosen element interpolation and from the choice of a Gaussian full integration scheme.

The components of $\underline{\underline{K}}$ coupling displacement and plastic slips read

$$
K_{u_{I} \gamma_{J}^{(\beta)}}=K_{\gamma_{J}^{(\beta)} u_{I}}=-2 v \mu \cos \left(2 \theta_{\beta}\right) \int_{-1}^{1} \frac{\mathrm{d} N_{I}(r(x))}{\mathrm{d} x} N_{J}(r) \frac{H_{\mathrm{el}}}{2} \mathrm{~d} r=-v \mu \cos \left(2 \theta_{\beta}\right) r_{I},
$$

where $r_{I}$ is the intrinsic coordinate of the node $I\left(r_{A}=-1, r_{B}=1\right)$.

The components of $\underline{\underline{K}}$ solely related to the slips read

$$
\begin{aligned}
K_{\gamma_{I}^{(\beta)} \gamma_{J}^{(\kappa)}=2 \int_{-1}^{1}}\left\{\left[\frac{\partial s^{(\beta)}}{\partial \Delta \gamma_{J}^{(\kappa)}}-\frac{\partial \sigma}{\partial \Delta \gamma_{J}^{(\kappa)}} \cos \left(2 \theta_{\beta}\right)\right] N_{I}(r)\right. \\
\left.+\left[\frac{\partial \mathrm{d}}{\partial \Delta \gamma_{J}^{(\kappa)}} \sin ^{2} \theta_{\beta}+\frac{\partial \omega^{(\beta)}}{\partial \Delta \gamma_{J}^{(\kappa)}}\right] \frac{\mathrm{d} N_{I}(r(x))}{\mathrm{d} x}\right\} \frac{H_{\mathrm{el}}}{2} \mathrm{~d} r .
\end{aligned}
$$




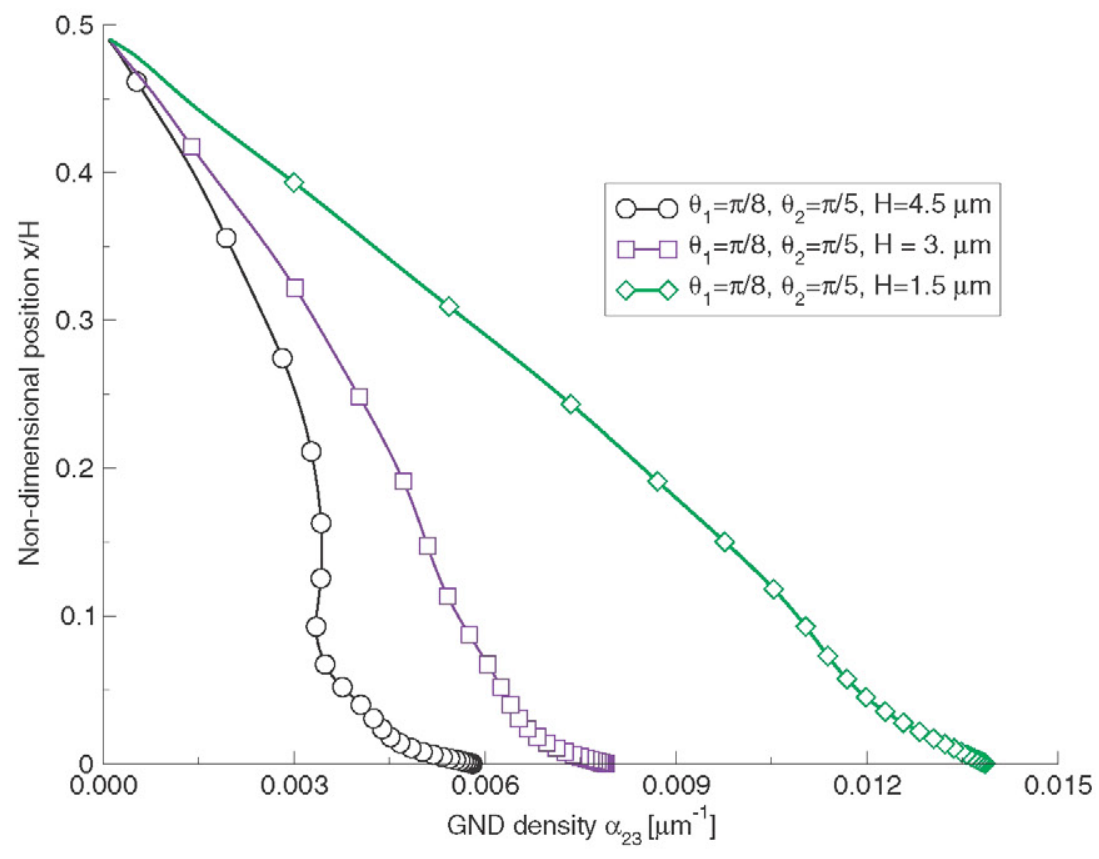

Figure 14. SGCP results in terms of GND density on a constrained crystalline strip endowed with two couples of symmetric slip systems, with applied shear $\Gamma=0.02$.

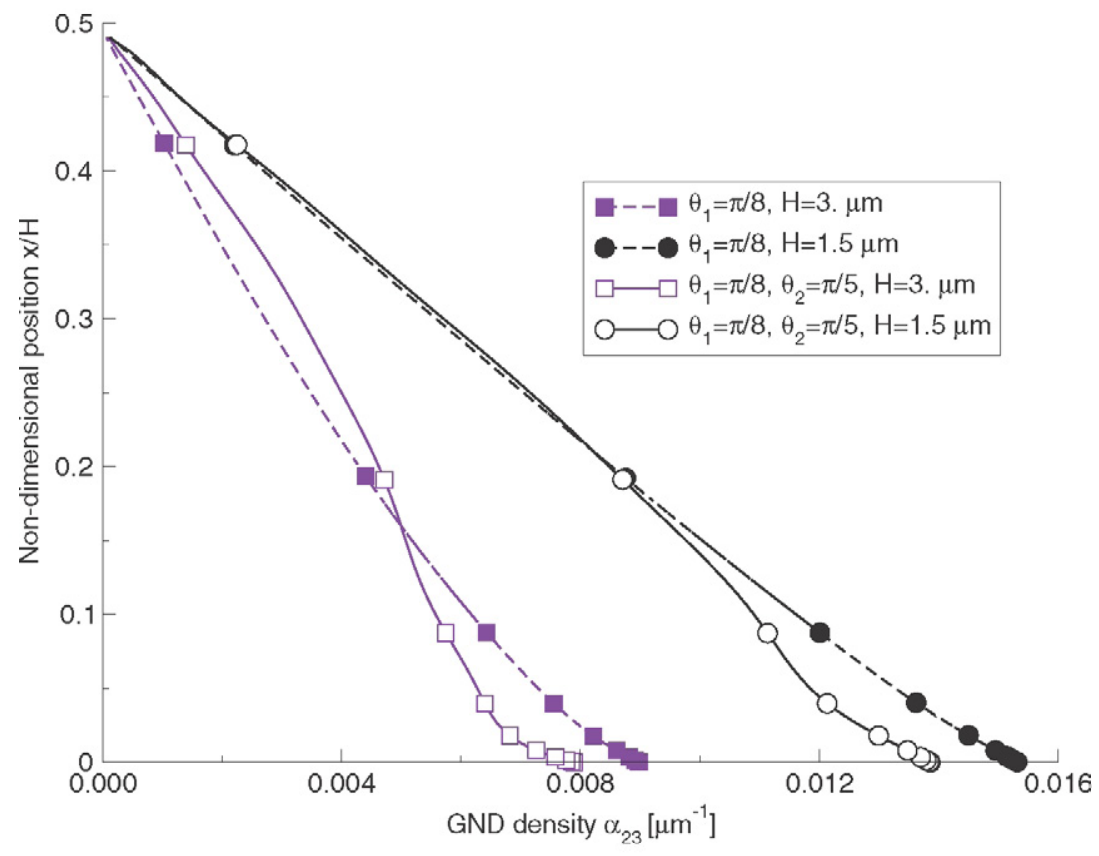

Figure 15. SGCP results in terms of GND density on a constrained crystalline strip endowed with either one couple or two couples of symmetric slip systems, with applied shear $\Gamma=0.02$. 
The relevant derivatives of the Cauchy and defect stresses are simple, so that we obtain

$$
\begin{aligned}
K_{\gamma_{I}^{(\beta)} \gamma_{J}^{(\kappa)}=\int_{-1}^{1}} & \left\{2 v \mu \left[\cos \left(2 \theta_{\beta}\right) \cos \left(2 \theta_{\kappa}\right) N_{I} N_{J}+\ell^{2} \sin ^{2} \theta_{\beta} \sin ^{2} \theta_{\kappa} \frac{\mathrm{d} N_{I}}{\mathrm{~d} x} \frac{\mathrm{d} N_{J}}{\mathrm{~d} x}\right.\right. \\
& \left.\times M\left\{2 \ell\left|\sum_{\delta=1}^{B}\left(\frac{\gamma_{t B}^{(\delta)}-\gamma_{t A}^{(\delta)}}{H_{\mathrm{el}}}+v \frac{\Delta \gamma_{B}^{(\delta)}-\Delta \gamma_{A}^{(\delta)}}{H_{\mathrm{el}}}\right) \sin ^{2} \theta_{\delta}\right|\right\}^{M-1}\right] \\
& \left.+\frac{\partial s^{(\beta)}}{\partial \Delta \gamma_{J}^{(\kappa)}} N_{I}+\frac{\partial \omega^{(\beta)}}{\partial \Delta \gamma_{J}^{(\kappa)}} \frac{\mathrm{d} N_{I}}{\mathrm{~d} x}\right\} H_{\mathrm{el}} \mathrm{d} r \\
= & 2 v \mu H_{\mathrm{el}}\left[\frac{1+\delta_{I J}}{3} \cos \left(2 \theta_{\beta}\right) \cos \left(2 \theta_{\kappa}\right)+2(-1)^{1+\delta_{I J}}\left(\frac{\ell}{H_{\mathrm{el}}}\right)^{2} \sin ^{2} \theta_{\beta} \sin ^{2} \theta_{\kappa}\right. \\
& \left.\times M\left\{2 \ell\left|\sum_{\delta=1}^{B}\left(\frac{\gamma_{t B}^{(\delta)}-\gamma_{t A}^{(\delta)}}{H_{\mathrm{el}}}+v \frac{\Delta \gamma_{B}^{(\delta)}-\Delta \gamma_{A}^{(\delta)}}{H_{\mathrm{el}}}\right) \sin ^{2} \theta_{\delta}\right|\right\}^{M-1}\right] \\
& +\int_{-1}^{1}\left(\frac{\partial s^{(\beta)}}{\partial \Delta \gamma_{J}^{(\kappa)}} N_{I}+\frac{\partial \omega^{(\beta)}}{\partial \Delta \gamma_{J}^{(\kappa)}} \frac{\mathrm{d} N_{I}}{\mathrm{~d} x}\right) H_{\mathrm{el}} \mathrm{d} r
\end{aligned}
$$

while

$$
\begin{aligned}
\frac{\partial \omega^{(\beta)}}{\partial \Delta \gamma_{I}^{(\kappa)}}= & \delta_{\beta \kappa} \frac{L_{\beta}^{2} \tau_{0}}{\left(\Delta t \gamma_{0}\right)^{N}}\{(N-1) \\
& \times\left[\left(\Delta \gamma^{(\beta)}\right)^{2}+\left(L_{\beta} \frac{\mathrm{d} \Delta \gamma^{(\beta)}}{\mathrm{d} x}\right)^{2}\right]^{\frac{N-3}{2}}\left(\Delta \gamma^{(\beta)} N_{I}(r)+L_{\beta}^{2} \frac{\mathrm{d} \Delta \gamma^{(\beta)}}{\mathrm{d} x} \frac{\mathrm{d} N_{I}(r(x))}{\mathrm{d} x}\right) \\
& \left.\times \frac{\mathrm{d} \Delta \gamma^{(\beta)}}{\mathrm{d} x}+\left[\left(\Delta \gamma^{(\beta)}\right)^{2}+\left(L_{\beta} \frac{\mathrm{d} \Delta \gamma^{(\beta)}}{\mathrm{d} x}\right)^{2}\right]^{\frac{N-1}{2}} \frac{\mathrm{d} N_{I}(r(x))}{\mathrm{d} x}\right\}
\end{aligned}
$$

and

$$
\begin{aligned}
\frac{\partial s^{(\beta)}}{\partial \Delta \gamma_{I}^{(\kappa)}}= & \delta_{\beta \kappa} \frac{g_{t}^{(\beta)}+v \Delta g^{(\beta)}}{\left(\Delta t \dot{\gamma}_{0}\right)^{N}}\{(N-1) \\
& \times\left[\left(\Delta \gamma^{(\beta)}\right)^{2}+\left(L_{\beta} \frac{\mathrm{d} \Delta \gamma^{(\beta)}}{\mathrm{d} x}\right)^{2}\right]^{\frac{N-3}{2}}\left(\Delta \gamma^{(\beta)} N_{I}+L_{\beta}^{2} \frac{\mathrm{d} \Delta \gamma^{(\beta)}}{\mathrm{d} x} \frac{\mathrm{d} N_{I}}{\mathrm{~d} x}\right) \Delta \gamma^{(\beta)} \\
& \left.+\left[\left(\Delta \gamma^{(\beta)}\right)^{2}+\left(L_{\beta} \frac{\mathrm{d} \Delta \gamma^{(\beta)}}{\mathrm{d} x}\right)^{2}\right]^{\frac{N-1}{2}} N_{I}\right\} \\
& +\frac{v}{\left(\Delta t \dot{\gamma}_{0}\right)^{N}}\left[\left(\Delta \gamma^{(\beta)}\right)^{2}+\left(L_{\beta} \frac{\mathrm{d} \Delta \gamma^{(\beta)}}{\mathrm{d} x}\right)^{2}\right]^{\frac{N-1}{2}} \Delta \gamma^{(\beta)} \frac{\partial \Delta g^{(\beta)}}{\partial \Delta \gamma_{I}^{(\kappa)}},
\end{aligned}
$$

where

$$
\begin{aligned}
\frac{\partial \Delta g^{(\beta)}}{\partial \Delta \gamma_{I}^{(\kappa)}}= & \Delta g^{(\beta)} \frac{-4 v h_{0}}{\tau_{s}-\tau_{0}} \tanh \frac{h_{0}\left(\lambda_{t}+v \Delta \lambda\right)}{\tau_{s}-\tau_{0}}\left[\frac{\Delta \gamma^{(\kappa)} N_{I}+L_{\kappa}^{2} \frac{\mathrm{d} \Delta \gamma^{(\kappa)}}{\mathrm{d} x} \frac{\mathrm{d} N_{I}}{\mathrm{~d} x}}{\Delta \gamma_{\mathrm{eff}}^{(\kappa)}}\right. \\
& \left.+\ell \sin ^{2} \theta_{\kappa} \frac{\mathrm{d} N_{I}}{\mathrm{~d} x} \sum_{\delta=1}^{B}\left(1-\delta_{\delta \kappa}\right) \operatorname{sgn}\left(\sum_{\omega=1}^{B}\left(1-\delta_{\omega \delta}\right) \frac{\mathrm{d} \Delta \gamma^{(\omega)}}{\mathrm{d} x} \sin ^{2} \theta_{\omega}\right)\right] \\
& +\mathcal{H}(\lambda)\left[\left[q+(1-q) \delta_{\beta_{k}}\right] \frac{\Delta \gamma^{(\kappa)} N_{I}+L_{\kappa}^{2} \frac{\mathrm{d} \Delta \gamma^{(\kappa)}}{\mathrm{d} x} \frac{\mathrm{d} N_{I}}{\mathrm{~d} x}}{\Delta \gamma_{\mathrm{eff}}^{(\kappa)}}\right.
\end{aligned}
$$




$$
\begin{aligned}
& +2\left(\dot{\epsilon}_{0} \Delta t\right)^{1-n_{p}} \ell^{n_{p}} n_{p} \sin ^{2} \theta_{\kappa} \frac{\mathrm{d} N_{I}}{\mathrm{~d} x} \\
& \left.\times\left(1-\delta_{\kappa \beta}\right)\left(\Delta \alpha^{(\beta)}\right)^{n_{p}-1} \operatorname{sgn}\left(\sum_{\omega=1}^{B}\left(1-\delta_{\omega \delta}\right) \frac{\mathrm{d} \Delta \gamma^{(\omega)}}{\mathrm{d} x} \sin ^{2} \theta_{\omega}\right)\right] .
\end{aligned}
$$

\section{References}

[1] Segurado J, Llorca J and Romero I 2007 Computational issues in the simulation of two-dimensional discrete dislocation dynamics. Modelling Simul. Mater. Sci. Eng. 15 S361-75

[2] Romero I, Segurado J and Llorca J 2008 Dislocation dynamics in non-convex domains using finite elements with embedded discontinuities Modelling Simul. Mater. Sci. Eng. 16035008

[3] Kubin L P, Canova G, Condat M, Devincre B, Pontikis V and Bréchet Y 1992 Dislocation microstructures and plastic flow: A 3D simulation Solid State Phenom. 23-24 455-72

[4] van der Giessen E and Needleman A 1995 Discrete dislocation plasticity: a simple planar model Modelling Simul. Mater. Sci. Eng. 3 689-735

[5] Bardella L 2006 A deformation theory of strain gradient crystal plasticity that accounts for geometrically necessary dislocations J. Mech. Phys. Solids $\mathbf{5 4} 128-60$

[6] Bardella L 2007 Some remarks on the strain gradient crystal plasticity modelling, with particular reference to the material length scales involved Int. J. Plasticity 23 296-322

[7] Bardella L and Giacomini A 2008 Influence of material parameters and crystallography on the size effects describable by means of strain gradient plasticity J. Mech. Phys. Solids 56 2906-34

[8] Gurtin M E and Anand L 2007 A gradient theory for single-crystal plasticity Modelling Simul. Mater. Sci. Eng. $15263-70$

[9] Gurtin M E, Anand L and Lele S P 2007 Gradient single-crystal plasticity with free energy dependent on dislocation densities J. Mech. Phys. Solids 55 1853-78

[10] Kuroda M and Tvergaard V 2008 On the formulations of higher-order strain gradient crystal plasticity models J. Mech. Phys. Solids 56 1591-608

[11] Peirce D, Asaro R J and Needleman A 1983 Material rate dependence and localized deformation in crystalline solids Acta Metall. 31 1951-76

[12] Borg U 2007 Strain gradient crystal plasticity effects on flow localization Int. J. Plasticity 23 1400-16

[13] Franciosi P, Berveiller M and Zaoui A 1980 Latent hardening in copper and aluminium single crystals Acta Metall. 28 273-83

[14] Havner K S 1992 Finite Plastic Deformation of Crystalline Solids (Cambridge Monographs on Mechanics and Applied Mathematics) (Cambridge: Cambridge University Press)

[15] Nye J F 1953 Some geometrical relations in dislocated crystals Acta Metall. 1 153-62

[16] Fleck N A and Hutchinson J W 1997 Strain gradient plasticity Adv. Appl. Mech. 33 295-361

[17] Gurtin M E 2002 A gradient theory of single-crystal viscoplasticity that accounts for geometrically necessary dislocations J. Mech. Phys. Solids 50 5-32

[18] Devincre B, Hoc T and Kubin L 2008 Dislocation mean free paths and strain hardening of crystals Science $3201745-8$

[19] ABAQUS User's \& Theory Manuals - Release 6.10-1 (Dassault Systémes: Providence, RI)

[20] Yefimov S, Groma I and van der Giessen E 2004 A comparison of a statistical-mechanics based plasticity model with discrete dislocation plasticity calculations J. Mech. Phys. Solids 52 279-300

[21] Evers L P, Brekelmans W A M and Geers M G D 2004 Non-local crystal plasticity model with intrinsic SSD and GND effects J. Mech. Phys. Solids 52 2379-401

[22] Bayley C J, Brekelmans W A M and Geers M G D 2006 A comparison of dislocation induced back stress formulations in strain gradient crystal plasticity Int. J. Solids Struct. 43 7268-86

[23] Geers M G D, Brekelmans W A M and Bayley C J 2007 Second-order crystal plasticity: internal stress effects and cyclic loading Modelling Simul. Mater. Sci. Eng. 15 S133-45

[24] Yalçinkaya T, Brekelmans W A M and Geers M G D 2012 Non-convex rate dependent strain gradient crystal plasticity and deformation patterning Int. J. Solids Struct. $492625-36$

[25] Ertürk I, van Dommelen J A W and Geers M G D 2009 Energetic dislocation interactions and thermodynamical aspects of strain gradient crystal plasticity theories J. Mech. Phys. Solids 57 1801-14

[26] Gurtin M E and Ohno N 2011 A gradient theory of small-deformation, single crystal plasticity that accounts for GND-induced interactions between slip systems J. Mech. Phys. Solids 59 320-43 
[27] Yefimov S, van der Giessen E and Groma I 2004 Bending of a single crystal: discrete dislocation and nonlocal crystal plasticity simulations Modelling Simul. Mater. Sci. Eng. 12 1069-86

[28] Bittencourt E, Needleman A, Gurtin M E and van der Giessen E 2003 A comparison of nonlocal continuum and discrete dislocation plasticity predictions J. Mech. Phys. Solids 51 281-310

[29] Nicola L, van der Giessen E and Gurtin M E 2005 Effect of defect energy on strain-gradient predictions of confined single-crystal plasticity J. Mech. Phys. Solids 53 1280-94

[30] Bargmann S, Svendsen B and Ekh M 2011 An extended crystal plasticity model for latent hardening in polycrystals Comput. Mech. $\mathbf{4 8} 631-45$

[31] Reddy B D, Wieners C and Wohlmuth B 2012 Finite element analysis and algorithms for single-crystal straingradient plasticity Int. J. Numer. Methods Eng. 90 784-804

[32] Gurtin M E, Fried E and Anand L 2010 The Mechanics and Thermodynamics of Continua (Cambridge: Cambridge University Press)

[33] Wulfinghoff S and Böhlke T 2012 Equivalent plastic strain gradient enhancement of single crystal plasticity: theory and numerics Proc. R. Soc. Lond. A $4682682-703$

[34] Shu J Y, King W E and Fleck N A 1999 Finite elements for materials with strain gradient effects Int. J. Numer. Methods Eng. 44 373-91

[35] Kuroda M 2011 On large-strain finite element solutions of higher-order gradient crystal plasticity Int. J. Solids Struct. 48 3382-94

[36] Ostien J and Garikipati K 2008 A discontinuous Galerkin method for an incompatibility-based strain gradient plasticity theory IUTAM Symp. Theoretical, Computational and Modelling Aspects of Inelastic Media (IUTAM Book Series vol 11) ed B D Reddy (Dordrecht: Springer) pp 217-26

[37] Gurtin M E 2004 A gradient theory of small-deformation isotropic plasticity that accounts for the Burgers vector and for dissipation due to plastic spin J. Mech. Phys. Solids $522545-68$

[38] Bardella L 2009 A comparison between crystal and isotropic strain gradient plasticity theories with accent on the role of the plastic spin Eur. J. Mech. A-Solid. 28 638-46

[39] Bardella L 2010 Size effects in phenomenological strain gradient plasticity constitutively involving the plastic spin Int. J. Eng. Sci. 48 550-68

[40] Segurado J and Llorca J 2009 An analysis of the size effect on void growth in single crystals using discrete dislocation dynamics Acta Mater. 57 1427-36

[41] Segurado J and Llorca J 2010 Discrete dislocation dynamics analysis of the effect of lattice orientation on void growth in single crystals Int. J. Plasticity 26 806-19

[42] Ashby M F 1970 The deformation of plastically non-homogeneous materials Phil. Mag. 21 399-424

[43] Arsenlis A and Parks D M 1999 Crystallographic aspects of geometrically-necessary and statistically-stored dislocation density Acta Mater: 47 1597-611

[44] Needleman A and Sevillano J G 2003 Preface to viewpoint set on: geometrically necessary dislocations and size dependent plasticity Scr: Mater. 48 109-11

[45] Kysar J W, Gan Y X, Morse T L, Chen X and Jones M E 2007 High strain gradient plasticity associated with wedge indentation into face-centered cubic single crystals: geometrically necessary dislocation densities $J$. Mech. Phys. Solids 55 1554-73

[46] Gurtin M E and Needleman A 2005 Boundary conditions in small-deformation, single-crystal plasticity that account for the Burgers vector J. Mech. Phys. Solids 53 1-31

[47] Evans A G and Hutchinson J W 2009 A critical assessment of theories of strain gradient plasticity Acta Mater: 57 1675-88

[48] Reddy B D 2011 The role of dissipation and defect energy in variational formulations of problems in straingradient plasticity: 1: Polycrystalline plasticity Continuum Mech Therm. 23 527-49

[49] Bardella L and Segurado J A comparison between strain gradient crystal plasticity and discrete dislocation dynamics Proc. AIMETA 2011: 20th Italian Conf. on Theoretical and Applied Mechanics (Bologna) ed F Ubertini et al

[50] Anand L, Gurtin M E, Lele S P and Gething C 2005 A one-dimensional theory of strain-gradient plasticity: formulation, analysis, numerical results J. Mech. Phys. Solids 53 1789-826

[51] Nicola L and van der Giessen E 2003 Discrete dislocation analysis of size effects in thin films J. Appl. Phys. $935920-8$

[52] Groma I, Györgyi G and Kocsis B 2007 Dynamics of coarse grained dislocation densities from an effective free energy Phil. Mag. 87 1185-99

[53] Ortiz M and Repetto E A 1999 Nonconvex energy minimization and dislocation structures in ductile single crystals J. Mech. Phys. Solids 47 397-462 